ANL-HEP-PR-98-27

JLAB-THY-98-11

\title{
Massive Lepton Pairs as a Prompt Photon Surrogate
}

\author{
Edmond L. Berger ${ }^{a}$, Lionel E. Gordon ${ }^{b, c}$, and Michael Klasen ${ }^{a}$ \\ ${ }^{a}$ High Energy Physics Division, Argonne National Laboratory \\ Argonne, Illinois 60439 \\ ${ }^{b}$ Jefferson Laboratory, Newport News, VA 23606 \\ ${ }^{c}$ Hampton University, Hampton, VA 23668
}

(March 16, 1998)

\begin{abstract}
We discuss the transverse momentum distribution for the production of massive lepton-pairs in hadron reactions at fixed target and collider energies within the context of next-to-leading order perturbative quantum chromodynamics. For values of the transverse momentum $Q_{T}$ greater than roughly half the pair mass $Q, Q_{T}>Q / 2$, we show that the differential cross section is dominated by subprocesses initiated by incident gluons. Massive leptonpair differential cross sections are an advantageous source of constraints on the gluon density, free from the experimental and theoretical complications of photon isolation that beset studies of prompt photon production. We compare calculations with data and provide predictions for the differential cross section as a function of $Q_{T}$ in proton-antiproton reactions at center-of-mass energies of $1.8 \mathrm{TeV}$, and in proton-nucleon reactions at fixed target and LHC energies.
\end{abstract}


12.38.Bx, 12.38.Qk, 13.85.Qk

Typeset using REVTEX 


\section{INTRODUCTION AND MOTIVATION}

Both massive lepton-pair production, $h_{1}+h_{2} \rightarrow \gamma^{*}+X ; \gamma^{*} \rightarrow l \bar{l}$, and prompt real photon production, $h_{1}+h_{2} \rightarrow \gamma+X$ are valuable probes of short-distance behavior in hadron reactions. In addition to the opportunities they offer for tests of perturbative quantum chromodynamics (QCD), the two reactions supply critical information on parton momentum densities. Massive lepton-pair production, commonly referred to as the Drell-Yan process [1], provided early confirmation of three colors and of the size of next-to-leading contributions to the cross section differential in the pair mass Q. The mass and longitudinal momentum (or rapidity) dependences of the cross section (integrated over the transverse momentum $Q_{T}$ of the pair) serve as laboratory for measurement of the antiquark momentum density, complementary to deep-inelastic lepton scattering from which one gains information of the sum of the quark and antiquark densities. Inclusive prompt real photon production is a source of essential information on the gluon momentum density. At lowest order in perturbation theory, the reaction is dominated at large values of the transverse momentum $p_{T}$ of the produced photon by the "Compton" subprocess, $q+g \rightarrow \gamma+q$. This dominance is preserved at higher orders, indicating that the experimental inclusive cross section differential in $p_{T}$ may be used to determine the density of gluons in the initial hadrons [2] 5].

There are notable similarities in the theoretical analyses of the two processes. At firstorder in the strong coupling strength, $\alpha_{s}$, the Compton subprocess and the annihilation subprocess $q+\bar{q} \rightarrow \gamma+g$ supply the transverse momentum of the directly produced prompt photons. Identical subprocesses, with the real $\gamma$ replaced by a virtual $\gamma^{*}$, are responsible at $\mathcal{O}\left(\alpha_{s}\right)$ for the transverse momentum of massive lepton-pairs. An important distinction, however, is that fragmentation subprocesses play a very important role in prompt real photon production at colliders energies. In these long-distance fragmentation subprocesses, the photon emerges from the fragmentation of a final parton, e.g., $q+g \rightarrow q+g$, followed by $q \rightarrow \gamma+X$

Another significant distinction is that interest in $h_{1}+h_{2} \rightarrow \gamma^{*}+X$ has been drawn most 
often to the domain in which the pair mass $Q$ is relatively large, justifying a perturbative treatment based on a small value of $\alpha_{s}(Q)$ and the neglect of inverse-power high-twist contributions (except near the edges of phase space). On the other hand, interest in prompt real photon production is directed to the region of large values of $p_{T}$ with small $\alpha_{s}\left(p_{T}\right)$. Interest in the transverse momentum $Q_{T}$ dependence of the massive lepton-pair production cross section has tended to be focussed on very small values of $Q_{T}$ where the cross section is largest. Fixed-order perturbation theory [6] is applicable for large $Q_{T}$, but it is inadequate at small $Q_{T}$, and all-orders resummation methods [7-11] have been developed to address the region $Q_{T}<<Q$.

In this paper we focus on the $Q_{T}$ distribution for $h_{1}+h_{2} \rightarrow \gamma^{*}+X$ in the region where $Q_{T}$ is greater than roughly half of the mass of the pair, $Q_{T}>Q / 2$. We present and discuss calculations carried out in next-to-leading order QCD at both fixed target and collider energies. We show that the differential cross section in this region is dominated by subprocesses initiated by incident gluons. Massive lepton-pair differential cross sections are therefore a valuable, heretofore perhaps overlooked, source of constraints on the gluon density. We compare calculations with data and provide predictions for the differential cross section as a function of $Q_{T}$ in proton-antiproton reactions at a center-of-mass energy of $1.8 \mathrm{TeV}$, in proton-nucleon reactions at fixed-target momenta and proton-proton reactions at LHC energies.

The requirement that both $Q$ be large and $Q_{T}>Q / 2$ may well be punishing in terms of experimental rate, especially at fixed-target momenta. However, as long as $Q_{T}$ is large, the perturbative requirement of small $\alpha_{s}\left(Q_{T}\right)$ can be satisfied without a large value of $Q$. We therefore explore and advocate the potential advantages of studies of $d^{2} \sigma / d Q d Q_{T}$ as a function of $Q_{T}$ for modest values of $Q, Q \sim 2 \mathrm{GeV}$, below the range of the traditional DrellYan region. To be sure, one must avoid the region in $Q$ of prominent resonance formation, e.g., the regions of the $\rho, \rho^{\prime}$, and $J / \psi$ resonances, among others. In addition, there are various backgrounds with which to contend at small $Q$ such as the contributions to the event rate from prompt decays of heavy flavors, e.g., $h_{1}+h_{2} \rightarrow c+\bar{c}+X ; c \rightarrow l+X$. These 
heavy flavor contributions may be estimated by direct computation [12] and/or bounded through experimental measurement of the like-sign-lepton distributions. Previous studies of the connection between massive lepton-pair production and prompt photon production are reported in Refs. [13] and [14].

As our calculations demonstrate, the $Q_{T}$ distribution of massive lepton pair production presents a valuable additional method for direct measurement of the gluon momentum distribution. This method makes good use of a significant data base at collider and fixed target energies. The method is similar in principle to the approach based on prompt photon production, but it avoids the experimental and theoretical complications of photon isolation that beset studies of prompt photon production.

In $e^{+} e^{-}$and in hadron-hadron reactions at collider energies, prompt photons are observed and their cross sections are measured only if the photons are relatively isolated in phase space. Isolation is required to reduce various hadronic backgrounds including those from the electromagnetic decay of mesons, e.g., $\pi^{o} \rightarrow 2 \gamma$. The essence of isolation is that a cone of half-angle $\delta$ is drawn about the direction of the photon's momentum, and the isolated cross section is defined for photons accompanied by less than a specified amount of hadronic energy in the cone, e.g., $E_{h}^{\text {cone }} \leq E_{\max }=\epsilon_{h} E_{\gamma} ; E_{\gamma}$ denotes the energy of the photon. Instead of $\delta$, the Fermilab collider groups use the variable $R=\sqrt{(\Delta \phi)^{2}+(\Delta \eta)^{2}}$, where $\Delta \eta$ and $\Delta \phi$ denote differences of rapidity and azimuthal angle variables. Theoretical predictions will therefore depend upon the additional parameters $\epsilon_{h}$ and $\delta$ (or $R$ ). The isolated cross section is not an inclusive cross section, and the usual factorization theorems for inclusive cross sections need not apply [15]. Isolation removes backgrounds, but it also reduces the signal. For example, it reduces the contribution from processes in which the photon emerges from the long-distance fragmentation of quarks and gluons, themselves produced in short-distance hard collisions.

The necessity to invoke phenomenological fragmentation functions and the existing infrared ambiguity of the isolated cross section in next-to-leading order leaves some question about the extent to which isolated prompt photon data may be used for fully quantitative 
determinations of the gluon density. It is desirable to investigate other physical processes for extraction of the gluon density that are free from these systematic uncertainties.

Fortunately, no isolation would seem necessary in the case of virtual photon production (and subsequent decay into a pair of muons) in typical collider or fixed target experiments. Muons are observed only after they have penetrated a substantial hadron absorber. Thus, any hadrons within a typical cone about the direction of the $\gamma^{*}$ will have been stopped, and the massive lepton-pair signal will be entirely inclusive. For this reason, we claim that the cross section for massive lepton-pair production at large $Q_{T}$ is an advantageous source of information on the gluon density.

In Sec. II, we review the formalism of the next-to-leading order calculations of the transverse momentum distribution for both prompt photon production and massive lepton-pair production, demonstrating the analytic similarities and differences. In Sec. III, we present numerical calculations that establish the regions of $Q_{T}$ in which the gluon initiated Compton subprocess dominates the differential cross section $d^{2} \sigma / d Q d Q_{T}$. Comparisons with data and predictions are provided in Sec. IV. Fixed-target data [16] are limited to the region $Q_{T}<Q$, but they are useful for establishing the rough value in $Q_{T} \simeq Q / 2$ above which resummation may be ignored. Collider data [17] extend to larger values of $Q_{T}$. Our conclusions are summarized in Sec. V.

\section{MASSIVE LEPTON PAIR PRODUCTION AND PROMPT PHOTON PRODUCTION AT NEXT-TO-LEADING ORDER}

In inclusive hadron interactions at collider energies, $h_{1}+h_{2} \rightarrow \gamma^{*}+X$ with $\gamma^{*} \rightarrow l \bar{l}$, lepton pair production proceeds through partonic hard-scattering processes involving initial-state light quarks $q$ and gluons $g$. In lowest-order QCD, at $\mathcal{O}\left(\alpha_{s}^{0}\right)$, the only partonic subprocess is $q+\bar{q} \rightarrow \gamma^{*}$. At $\mathcal{O}\left(\alpha_{s}\right)$, both $q+\bar{q} \rightarrow \gamma^{*}+g$ and $q+g \rightarrow \gamma^{*}+q$ participate, with the recoil of the final parton balancing the transverse momentum of the lepton-pair. These processes

are shown in Figs. 1(a) and 2(a). Calculations of the cross section at order $\mathcal{O}\left(\alpha_{s}^{2}\right)$ involve 
virtual gluon loop corrections to these $\mathcal{O}\left(\alpha_{s}\right)$ subprocesses (Figs. 1(b) and 2(b)) as well as real gluon radiation contributions from a wide range of $2 \rightarrow 3$ parton subprocesses (of which some examples are shown in Figs. 1(c) and 2(c)).

The physical cross section is obtained through the factorization theorem,

$$
\frac{d^{2} \sigma_{h_{1} h_{2}}^{\gamma^{*}}}{d Q_{T}^{2} d y}=\sum_{i j} \int d x_{1} d x_{2} f_{h_{1}}^{i}\left(x_{1}, \mu_{f}^{2}\right) f_{h_{2}}^{j}\left(x_{2}, \mu_{f}^{2}\right) \frac{s d^{2} \hat{\sigma}_{i j}^{\gamma^{*}}}{d t d u}\left(s, Q, Q_{T}, y ; \mu_{f}^{2}\right) .
$$

It depends on the hadronic center-of-mass energy $S$ and on the mass $Q$, the transverse momentum $Q_{T}$, and the rapidity $y$ of the virtual photon; $\mu_{f}$ is the factorization scale of the scattering process. The usual Mandelstam invariants in the partonic system are defined by $s=\left(p_{1}+p_{2}\right)^{2}, t=\left(p_{1}-p_{\gamma^{*}}\right)^{2}$, and $u=\left(p_{2}-p_{\gamma^{*}}\right)^{2}$, where $p_{1}$ and $p_{2}$ are the momenta of the initial state partons and $p_{\gamma^{*}}$ is the momentum of the virtual photon. The indices $i j \in\{q \bar{q}, q g\}$ denote the initial parton channels whose contributions are added incoherently to yield the total physical cross section. The partonic cross section $\hat{\sigma}_{i j}^{\gamma^{*}}\left(s, Q, Q_{T}, y ; \mu_{f}^{2}\right)$ is obtained commonly from fixed-order QCD calculations through

$$
\frac{d^{2} \hat{\sigma}_{i j}^{\gamma^{*}}}{d t d u}=\alpha_{s}\left(\mu^{2}\right) \frac{d^{2} \hat{\sigma}_{i j}^{\gamma^{*},(a)}}{d t d u}+\alpha_{s}^{2}\left(\mu^{2}\right) \frac{d^{2} \hat{\sigma}_{i j}^{\gamma^{*},(b)}}{d t d u}+\alpha_{s}^{2}\left(\mu^{2}\right) \frac{d^{2} \hat{\sigma}_{i j}^{\gamma^{*},(c)}}{d t d u}+\mathcal{O}\left(\alpha_{s}^{3}\right) .
$$

The tree, virtual loop, and real emission contributions are labeled (a), (b), and (c) as are the corresponding diagrams in Figs. 1 and 2. The parameter $\mu$ is the renormalization scale. It is set equal to the factorization scale $\mu_{f}=\sqrt{Q^{2}+Q_{T}^{2}}$ throughout this paper.

The cross section for $h_{1}+h_{2} \rightarrow l \bar{l}+X$, differential in the invariant mass of the lepton pair $Q^{2}$ as well as its transverse momentum and rapidity, is obtained from Eq. (1) by the relation

$$
\frac{d^{3} \sigma_{h_{1} h_{2}}^{l \bar{l}}}{d Q^{2} d Q_{T}^{2} d y}=\left(\frac{\alpha_{e m}}{3 \pi Q^{2}}\right) \frac{d^{2} \sigma_{h_{1} h_{2}}^{\gamma^{*}}}{d Q_{T}^{2} d y}\left(S, Q, Q_{T}, y\right)
$$

where $Q^{2}=\left(p_{l}+p_{\bar{l}}\right)^{2}$, and $p_{l}, p_{\bar{l}}$ are the four-momenta of the two final leptons. The socalled Drell-Yan factor $\alpha_{e m} /\left(3 \pi Q^{2}\right)$ is included in all the numerical results for virtual photon production presented in Secs. III and IV.

As stated in Sec. I, the partonic subprocesses that contribute to lepton pair production and to prompt photon production at finite transverse momentum are identical except for the 
fact that the photon is off-shell in the case of massive lepton pair production. As expected, the $\mathcal{O}\left(\alpha_{s}\right)$ cross section formulæ for virtual photon production reduce to those for real photon production in the limit $Q^{2} \rightarrow 0$ [13]. It was also noted earlier that fragmentation processes, which are not present in the Drell-Yan case, are important for real photon production. Taking into account direct and fragmentation pieces, we express the inclusive cross section for prompt photon production $h_{1}+h_{2} \rightarrow \gamma+X$ as

$$
\begin{aligned}
\frac{d^{2} \sigma_{h_{1} h_{2}}^{\gamma}=}{d p_{T}^{2} d y}= & \sum_{i j} \int d x_{1} d x_{2} f_{h_{1}}^{i}\left(x_{1}, \mu_{f}^{2}\right) f_{h_{2}}^{j}\left(x_{2}, \mu_{f}^{2}\right) \\
& {\left[\frac{s d \hat{\sigma}_{i j}^{\gamma} \text { direct }}{d t d u}\left(s, p_{T}, y ; \mu_{f}^{2}, \mu_{F}^{2}\right)+\sum_{k} \int \frac{d z}{z^{2}} D_{k}^{\gamma}\left(z, \mu_{F}^{2}\right) \frac{s d \hat{\sigma}_{i j}^{k, f r a g m}}{d t d u}\left(s, p_{T}, y ; \mu_{f}^{2}, \mu_{F}^{2}\right)\right] . }
\end{aligned}
$$

The transverse momentum of the real photon is $p_{T}$. The fragmentation function $D_{k}^{\gamma}\left(z, \mu_{F}^{2}\right)$ is the probability for emission of a photon with momentum fraction $z$ from parton $k$ at the fragmentation scale $\mu_{F}^{2}$. The function is a non-perturbative quantity that cannot be predicted by QCD but instead requires input from experiment at some reference scale. Its evolution to greater values of the scale is specified by perturbation theory. In this paper, we set $\mu_{F}=\mu_{f}=\mu$.

In prompt photon production, fragmentation contributes at leading order $\mathcal{O}\left(\alpha_{s}\right)$. Although the hard subprocess matrix elements $\hat{\sigma}_{i j}^{k,}$ fragm in the fragmentation case are $\mathcal{O}\left(\alpha_{s}^{2}\right)$, the fragmentation function has a logarithmic dependence on the scale $\mu_{F}^{2}$ and is effectively of $\mathcal{O}\left(1 / \alpha_{s}\right)$. When the subprocess matrix elements are convoluted with the fragmentation function, the product is of $\mathcal{O}\left(\alpha_{s}\right)$.

At next-to-leading order $\mathcal{O}\left(\alpha_{s}^{2}\right)$, one must calculate virtual loop corrections to the leading order processes (Figs. 1(b) and 2(b)) and the three-body processes $q+g \rightarrow \gamma^{*}+q+g$, $q+\bar{q} \rightarrow \gamma^{*}+g+g, q+q \rightarrow \gamma^{*}+q+q, g+g \rightarrow \gamma^{*}+q+\bar{q}, q+\bar{q} \rightarrow \gamma^{*}+q+\bar{q}$, $q+q^{\prime} \rightarrow \gamma^{*}+q^{\prime}+q, q+\bar{q}^{\prime} \rightarrow \gamma^{*}+q^{\prime}+\bar{q}^{\prime}$, and $q+\bar{q} \rightarrow \gamma^{*}+q^{\prime}+\bar{q}^{\prime}$ (Figs. 1(c) and 2(c)). The $2 \rightarrow 3$ parton matrix elements are integrated in $4-2 \epsilon$ dimensions over the singular regions of phase space to expose the infrared and collinear singularities as $1 / \epsilon$-poles. These poles are then canceled against the poles present in the virtual corrections. In general, this procedure 
applies to virtual photons as well as real photons. If the limit $Q^{2} \rightarrow 0$ is taken carefully, and no overall powers of $Q^{2}$ are factored out, the results for the virtual photon cross section will again reduce to those for the real photon cross section, as they do in leading order.

There is, however, one particular case that has to be treated separately. In a next-toleading order calculation of prompt photon production, a singularity is produced whenever the photon becomes collinear to a final state quark in the integration of the matrix elements over phase-space. This collinear singularity must be subtracted and absorbed into the fragmentation function for a quark to produce a photon. The subtraction is done at an arbitrary fragmentation scale $\mu_{F}$, and hence the direct photon cross section acquires a logarithmic dependence on the scale $\mu_{F}$ that is partially canceled when fragmentation contributions are included. Fragmentation contributions are not present in the virtual photon case since the photon is off-shell, and its invariant mass $Q$ regulates the singularity. This finite mass regularization leads to a logarithmic dependence of the Drell-Yan cross section on the virtuality of the photon or the invariant mass of the observed lepton-pair $Q$. As long as $Q$ is large enough, these logarithms are small so that no resummation/factorization is necessary for a reliable prediction of the cross section. The Drell-Yan cross section will not have a fragmentation contribution except in the limit of very small $Q$.

As an example of the discussion in the paragraph above, we consider the subprocess $q \bar{q} \rightarrow q^{\prime} \bar{q}^{\prime} \gamma\left(\gamma^{*}\right)$ for prompt photon production and for the Drell-Yan case. The relevant diagrams are shown in Fig. 3(a) and 3(b) for initial- and final-state photon radiation. Since we are interested mainly in the structure of the final state in the region where a photon becomes collinear to a quark, this subprocess is typical and any conclusions drawn from it can be applied to the more complicated processes such as $q g$ scattering. In this discussion we concentrate on the final-state collinear regions, but we use the full three-body matrix elements for both Drell-Yan [18] and prompt photons [5] integrated fully over phase space, before factorization of any singularities. The exact forms for the phase space integrals can be found in Ref. [5] for prompt photons and in Refs. [19, [18] for the Drell-Yan case. For the 
direct photon cross section $s d^{2} \hat{\sigma}_{i j}^{\gamma}$, direct $/ d t d u$, the phase space integrals yield a singular term of the form

$$
\frac{1}{\epsilon} 2 K_{\gamma} \frac{-\left(t^{2}+u^{2}\right)\left(2 s^{2}+2 s t+t^{2}+2 s u+2 t u+u^{2}\right)}{s(t+u)^{4}}=\frac{1}{\epsilon} f(s, t, u)
$$

whereas the corresponding logarithmic term in the Drell-Yan case is

$$
\begin{aligned}
& \ln \left[\frac{s+Q^{2}-s_{2}+\lambda}{s+Q^{2}-s_{2}-\lambda}\right] K_{D Y} \\
& {\left[\frac{1}{\lambda^{5}}\left(\frac{3 Q^{2} u^{2}(u-t)\left(t+u-2 s_{2}\right)}{s}+\frac{3\left(s-Q^{2}\right) u^{2}\left(u^{2}-t^{2}\right)}{Q^{2}+s-s_{2}}\right)\right.} \\
& +\frac{1}{\lambda^{3}}\left(\frac{u\left(2 s s_{2}-2 s_{2}^{2}+2 s_{2} t-s u+4 s_{2} u-t u-3 u^{2}\right)}{s}\right. \\
& \left.+\frac{u^{2}}{\lambda}\left(\frac{1}{Q^{2}+s-s_{2}}\left(\frac{3 s}{4}-\frac{s_{2}}{2}+u\right)+\frac{1}{Q^{2}+s-s_{2}}\left(\frac{3 s}{2}+\frac{5 u}{2}+\frac{2 u^{2}}{s}\right)\right)\right] \\
& \left.\left.+(t \longleftrightarrow u)+3 u-\frac{u^{2}}{s}\right)\right) \\
& =\ln \left[\frac{s+Q^{2}-s_{2}+\lambda}{s+Q^{2}-s_{2}-\lambda}\right] g\left(s, t, u, Q^{2}\right) .
\end{aligned}
$$

The quantities $K_{\gamma}$ and $K_{D Y}$ represent products of color, electric charge, and phase space factors. The function $\lambda=\sqrt{(t+u)^{2}-4 Q^{2} s_{2}} ; s, t$, and $u$ are defined just below Eq. (11), and $s_{2}=s+t+u-Q^{2}$.

The functions $f(s, t, u)$ and $g\left(s, t, u, Q^{2}\right)$ differ, of course, due to the $Q^{2}$-dependence in the Drell-Yan case. In the limit $Q^{2} \rightarrow 0$, however, $g\left(s, t, u, Q^{2}\right) \rightarrow f(s, t, u)$, as expected. The logarithm in Eq. (6) becomes infinite in this limit and thus corresponds to the pole 1/ $\epsilon$. For the prompt photon case the pole term is subtracted in some factorization scheme by the addition of a counter term. In the $\overline{\mathrm{MS}}$ scheme the counter term is schematically

$$
K(\epsilon) \frac{1}{\epsilon}\left(\frac{\mu^{2}}{\mu_{F}^{2}}\right)^{\epsilon} \hat{\sigma}^{q \bar{q} \rightarrow q^{\prime} \bar{q}^{\prime}} P_{\gamma / q}(z)
$$

where $\hat{\sigma}^{\bar{q} q}$ is the hard 2-body subprocess matrix element, and $P_{\gamma / q}(z)$ is the usual splitting function to produce a photon from a quark. This procedure replaces the pole term with new terms depending on $\ln \left(\mu_{F}^{2}\right)$. 
To summarize this Section, we have shown that the cross section formulæ for virtual and real photon production in hadronic collisions are intimately related in leading order $\mathcal{O}\left(\alpha_{s}\right)$ as well as in next-to-leading order $\mathcal{O}\left(\alpha_{s}^{2}\right)$. If the logarithmic terms in $Q^{2}$ are first isolated, setting $Q^{2}=0$ in the rest of the Drell-Yan cross section leads to a recovery of the prompt photon cross section except for the $\ln \left(\mu_{F}^{2}\right)$ terms and numerically small finite terms associated with the factorization of the collinear photon singularity into the fragmentation function. The situation is completely analogous to hard photoproduction where the photon participates in the scattering in the initial state instead of the final state. For real photons, one encounters an initial-state singularity that is factorized into a photon structure function. For virtual photons, this singularity is replaced by a logarithmic dependence on the photon virtuality $Q$ [20].

\section{DOMINANCE OF THE COMPTON SUBPROCESSES FOR $Q_{T}>Q / 2$}

Having identified in Sec. II the subprocesses that contribute in next-to-leading order to massive lepton-pair production, $h_{1}+h_{2} \rightarrow \gamma^{*}+X$, and to prompt real photon production, $h_{1}+h_{2} \rightarrow \gamma+X$, and having established the correspondence between the analytic forms of the contributions to these two reactions, we turn in this Section to explicit evaluations of the differential cross sections as functions of $Q_{T}$ and $p_{T}$, respectively, at both fixed-target momenta and collider energies. We work in the $\overline{\mathrm{MS}}$ renormalization scheme and set the renormalization and factorization scales equal. We employ the CTEQ4M set of parton densities [21] and a two-loop expression for the strong coupling strength $\alpha_{s}(\mu)$, with five flavors and appropriate threshold behavior at $\mu=m_{b}$. For the massive lepton-pair case, $\alpha_{s}$ is evaluated at a hard scale $\mu=\sqrt{Q^{2}+Q_{T}^{2}}$, and for prompt real photon production, $\mu=p_{T}$.

Beginning with $p+N \rightarrow \gamma^{*}+X$ at $p_{l a b}=800 \mathrm{GeV}$, we present the invariant inclusive cross section $E d^{3} \sigma / d p^{3}$ as a function of $Q_{T}$ in Fig. 4. Shown in this figure are the $q \bar{q}$ and $q g$ perturbative contributions to the cross section at leading order and at next-to-leading order. For this calculation, we integrate the invariant inclusive cross section over the scaled 
longitudinal momentum range $0.1<x_{F}<0.3$ and over the mass interval $5<Q<6 \mathrm{GeV}$, and we divide by the bin width in $x_{F}$. To represent the target nucleon $\mathrm{N}$, we use parton densities equal to $50 \%$ of the proton densities and $50 \%$ of the neutron densities, having in mind a comparison with deuterium data from Fermilab experiment E772, to be discussed in Sec. IV.

The results in Fig. 4 show that the leading-order and the next-to-leading order contributions are about equal in size for $Q_{T}>1 \mathrm{GeV}$ in both the $q \bar{q}$ and $q g$ channels, meaning that the cross section through next-to-leading order is about twice that of the leading-order value. For $Q_{T}<2 \mathrm{GeV}$, the $q \bar{q}$ contribution exceeds that of $q g$ channel. However, for values of $Q_{T}>2 \mathrm{GeV}$, the $q g$ contribution becomes increasingly important. As shown in Fig. 5(a), the $q g$ contribution accounts for about $80 \%$ of the rate once $Q_{T} \simeq Q$. The results in Fig. 5(a) also demonstrate that subprocesses other than those initiated by the $q \bar{q}$ and $q g$ initial channels are of negligible import.

In Fig. 5(b), we display the fractional contributions to the cross section as a function of $Q_{T}$ for a larger value of $\mathrm{Q}: 11<Q<12 \mathrm{GeV}$. In this case, the fraction of the rate attributable to $q g$ initiated subprocesses again increases with $Q_{T}$. It becomes $80 \%$ or greater for $Q_{T} \simeq Q / 2$.

For the calculations reported in Figs. 4 and 5(a,b), we chose values of $\mathrm{Q}$ in the traditional range for studies of massive lepton-pair production, viz., above the interval of the $J / \psi$ and $\psi^{\prime}$ states and either below or above the interval of the $\Upsilon^{\prime} s$.

For Fig. 5(c), we select the interval $2.0<Q<3.0 \mathrm{GeV}$. In this region, one would be inclined to doubt the reliability of leading-twist perturbative descriptions of the cross section $d \sigma / d Q$, integrated over all $Q_{T}$. However for values of $Q_{T}$ that are large enough, a perturbative description of the $Q_{T}$ dependence of $d^{2} \sigma / d Q d Q_{T}$ ought to be justified. The results presented in Fig. 5(c) demonstrate that, as at higher masses, the $q g$ incident subprocesses dominate the cross section for $Q_{T}$ larger than a few $\mathrm{GeV}$.

We return to $p N$ reactions at fixed-target momenta in Sec. IV and discuss comparisons of our cross sections with data, but we address first the case of $p+\bar{p} \rightarrow \gamma^{*}+X$ at collider 
energies.

In Fig. 6, we display the theoretical invariant inclusive cross section at the energy of the FNAL collider energy as a function of $Q_{T}$ for four regions of $Q$. In addition to the regions of $Q$ mentioned above, we select a region of relatively large $Q, 30.0<Q<35.0 \mathrm{GeV}$, to extend the region of interest of our analysis. For these results at collider energies, we average over the rapidity interval $-1.0<y<1.0$. The fractional contributions from the $q g$ and $q \bar{q}$ subprocesses up through next-to-leading order are shown in Fig. 7. Evident in Fig. 7 is that the $q g$ subprocess is the most important subprocess as long as $Q_{T}>Q / 2$. The dominance of the $q g$ subprocess diminishes somewhat with $Q$, dropping from over $80 \%$ for the lowest values of $Q$ to about $70 \%$ at its maximum for $Q \simeq 30 \mathrm{GeV}$. In addition, for very large $Q_{T}$, the significant luminosity associated with the valence dominated $\bar{q}$ density in $p \bar{p}$ reactions begins to raise the fraction of the cross section attributed to the $q \bar{q}$ subprocesses.

The calculations presented in Figs. 4 - 7 show convincingly that data on the transverse momentum dependence of the cross section for massive lepton-pair production at both fixedtarget and collider energies should be a very valuable independent source of information on the gluon density. It is instructive to compare the results at collider energies with those expected for prompt real photon production. In Fig. 8, we present the predicted differential cross section for prompt photon production in the extreme cases: (a) all contributions, with full fragmentation taken into consideration (solid line), and (b) no fragmentation contributions included (dashed line). The breakdown of these two cross sections into their $q \bar{q}$ and $q g$ components is presented in Fig. 9. Comparing the magnitudes of the prompt photon and massive lepton pair production cross sections in Figs. 6 and 8, we note that the inclusive prompt photon cross section is about a factor of 400 greater than the massive lepton-pair cross section integrated over the mass interval $2.0<Q<3.0 \mathrm{GeV}$. This factor is attributable in large measure to the factor $\alpha_{e m} /\left(3 \pi Q^{2}\right)$ associated with the decay of the virtual photon to $\mu^{+} \mu^{-}$. At current integrated luminosities, prompt photons have been observed with values of $p_{T}$ extending to $100 \mathrm{GeV}$ and beyond [23]. It appears that it should be possible to examine massive lepton-pair cross sections in the same data sample out to $Q_{T}$ of $30 \mathrm{GeV}$ 
or more. As may be appreciated from a comparison of Figs. 7 and 9, dominance of the $q g$ contribution in the massive lepton-pair case is as strong if not stronger than it is in the prompt photon case. The statistical limitation to $Q_{T}$ of $30 \mathrm{GeV}$ or so in the current data sample means that the reach in $x_{\text {gluon }}$, the fractional light-cone momentum carried by the incident gluon, is limited presently to $2 Q_{T} / \sqrt{S} \sim 0.033$ or so, about a factor of three less than that potentially accessible with prompt photons in the same sample of data. It is valuable to be able to investigate the gluon density in the region $x_{\text {gluon }} \sim 0.033$, and less, with a process that has reduced experimental and theoretical systematic uncertainties from those of the prompt photon case.

\section{COMPARISONS WITH DATA AND PREDICTIONS}

In this Section we compare our calculations with data on massive lepton-pair production at large values of $Q_{T}$, try to establish the domain in $Q_{T}$ over which the next-to-leading order perturbative calculations should be reliable, and offer predictions in addition to those presented in Sec. III.

In Fig. 10, we show the invariant inclusive cross section $E d^{3} \sigma / d p^{3}$ as a function of $Q_{T}$ for $p+\bar{p} \rightarrow \gamma^{*}+X$, with $\gamma^{*} \rightarrow \mu^{+} \mu^{-}$, at $\sqrt{S}=630 \mathrm{GeV}$, with $2 m_{\mu}<Q<2.5 \mathrm{GeV}$. These results are averaged over the rapidity interval $-1.7<y<1.7$. We present the next-toleading order perturbative cross section along with its two major components, the $q g$ and $q \bar{q}$ contributions. The theoretical expectation is in good agreement with the data published by the CERN UA1 collaboration [17]. Dominance of the $q g$ component is evident over a large interval in $Q_{T}$, whereas the $q \bar{q}$ contribution begins to be felt at very large $Q_{T}$.

Data obtained by the Fermilab E772 collaboration [16,22] from an $800 \mathrm{GeV}$ proton beam incident on a deuterium target are shown in Fig. 11 along with theoretical calculations. The solid lines show the purely perturbative next-to-leading order expectation, and the dashed and dotted curves pertain to results obtained from all-orders soft-gluon resummation and its perturbative expansion at low $Q_{T}$. 
The leading-order $\mathcal{O}\left(\alpha_{s}\right)$ and next-to-leading order $\mathcal{O}\left(\alpha_{s}^{2}\right)$ perturbative calculations of the $Q_{T}$ distribution necessarily fail for $Q_{T}<<Q$, where the analytic form of the fixed-order cross section behaves as

$$
d \sigma / d Q_{T}^{2} \sim \alpha_{s} / Q_{T}^{2}\left\{1+a_{1} \alpha_{s} \ln ^{2}\left(Q / Q_{T}\right)+a_{2} \alpha_{s}^{2} \ln ^{4}\left(Q / Q_{T}\right)+\ldots\right\},
$$

In Eq. (8), $\alpha_{s}(\mu)$ is evaluated at the scale $\mu=Q$, and the $a_{i}$ are coefficients that do not depend on $Q_{T}$. The expansion parameter of the series in Eq. (8) is $\alpha_{s} \ln ^{2}\left(Q / Q_{T}\right)$, not $\alpha_{s}$, and it can become large at small $Q_{T}$. The logarithmic series, associated with soft-gluon emission, is a vestige of the singularities that cancel at each order in perturbation theory between the real gluon emission and virtual gluon exchange diagrams. An elaborate procedure exists to sum the logarithmic series, known as resummation [7 [1]. We employ the formulation published by Arnold and Kauffman [10], along with the phenomenological non-perturbative functions of Ladinsky and Yuan [11]. We compare the resummed results with the purely perturbative results in order to try to establish the value above which we can be reasonably confident that fixed-order perturbation theory is adequate on its own.

In Fig. 11, the dashed curves show the theoretical cross section obtained from resummation. They are applicable only in the region $Q_{T}<<Q$. This restricted region of applicability arises because the only part of the fixed-order perturbative cross sections that is incorporated in the all-orders resummation formalism is the part that diverges as $Q_{T}^{-2}$ when $Q_{T} \rightarrow 0$. The dotted curves in Fig. 11 show what is termed the "asymptotic" fixed-order contribution. It is obtained from an expansion of the resummed cross section in a power series in $\alpha_{s}(Q)$. Mathematically, it represents the divergent part of the fixed-order perturbative cross section asymptotically in the limit $Q_{T} \rightarrow 0$, and, as the coincidence of the dotted and solid curves shows in Fig. 11, this representation is exact at very small $Q_{T}$. In our case, the dotted curves are the asymptotic answer through $\mathcal{O}\left(\alpha_{s}^{2}\right)$.

Whereas resummation is clearly needed at small $Q_{T}$, a fixed-order perturbative calculation, without resummation, should be adequate at large enough $Q_{T}$. In addition to the singular parts incorporated in the resummation, fixed-order perturbative cross sections in- 
clude remainders that are non-singular at small $Q_{T}$ but are important at large $Q_{T}$. An issue addressed in the literature is how best to connect the region in which all-orders resummation is pertinent to the region in which the simpler fixed-order result is fine. In efforts to restore the full content of fixed-order cross sections in the limit of large $Q_{T}$, proposals have been made whereby the non-singular parts are reintroduced through "matching" prescriptions. The results of one approach to matching are sketched in Fig. 11 as the dot-dashed lines. The resummed result at very small $Q_{T}$ is joined smoothly to the purely perturbative fixed-order result at large $Q_{T}$.

Our purpose is to establish where the data on massive lepton-pair production may be used to test fixed-order perturbative QCD and to provide new constraints on the gluon density. The region of small $Q_{T}$ and the matching region of intermediate $Q_{T}$ are complicated by some level of phenomenological ambiguity. Within the CSS resummation approach, phenomenological non-perturbative functions play a key role in fixing the shape of the $Q_{T}$ spectrum at very small $Q_{T}$, and matching methods in the intermediate region are hardly unique. For the goals we have in mind, it would appear best to restrict attention to the region in $Q_{T}$ above the value at which the resummed result (dashed curves in Fig. 11) falls below the fixed-order perturbative expectation (solid curves). A rough rule-of-thumb based on the calculations shown in Fig. 11 is $Q_{T} \geq Q / 2$. The published data [16,22] at fixedtarget momenta shown in Figs. 11(a) and 11(b) do not extend into this domain. Their reach is exhausted at just about the value in $Q_{T}$ at which the fixed-order calculation should be reliable.

To establish the utility of massive lepton-pair production at fixed-target momenta for extraction of the gluon density, it would be valuable to obtain data in the traditional Drell-Yan region above $Q \simeq 4.0 \mathrm{GeV}$ but whose reach in $Q_{T}$ extends into the fixed-order perturbative domain, as defined above. The massive lepton-pair production cross section increases as $Q$ is reduced. In Fig. 11(c) we show the predicted cross section as a function of $Q_{T}$ for 2.0 $<Q<3.0 \mathrm{GeV}$. The region $Q_{T} \geq 3.0 \mathrm{GeV}$ is inviting. It is above the region in which resummation need be considered, and as shown in Fig. 5(c), it is a region in which the $q g$ 
subprocess dominates the final cross section.

In Fig. 12, we provide predictions of the the invariant inclusive cross section $E d^{3} \sigma / d p^{3}$ as a function of $Q_{T}$ for $p+p \rightarrow \gamma^{*}+X$ at the LHC energy $\sqrt{S}=14 \mathrm{TeV}$. The curves show the next-to-leading order perturbative predictions integrated over four different intervals of $\mathrm{Q}$ and averaged over the rapidity interval $-1.0<y<1.0$. In all four cases, the contribution from the $q g$ channel dominates at the level of $80 \%$ or greater over the range of $Q_{T}$ shown. The LHC cross sections are about an order of magnitude greater than those at $\sqrt{S}=1.8 \mathrm{TeV}$ over the range of $Q_{T}$ shown.

\section{DISCUSSION AND CONCLUSIONS}

Prompt real photon production is a source of essential information on the gluon momentum density. At lowest order in perturbation theory, the reaction is dominated at large values of the transverse momentum $p_{T}$ of the produced photon by the "Compton" subprocess, $q+g \rightarrow \gamma+q$. This dominance by gluon initiated subprocesses is preserved at higher orders, indicating that the experimental inclusive cross section differential in $p_{T}$ may be used to determine the density of gluons in the initial hadrons. There are notable similarities in the theoretical analyses of the massive lepton-pair production, the Drell-Yan process and prompt photon production. At first-order in the strong coupling strength, $\alpha_{s}$, the Compton subprocess and the annihilation subprocess $q+\bar{q} \rightarrow \gamma+g$ supply the transverse momentum of the photons in both cases. The parallels are maintained in next-to-leading order, as summarized in Sec. II.

In this paper we focus on the $Q_{T}$ distribution for $h_{1}+h_{2} \rightarrow \gamma^{*}+X$. We present and discuss calculations carried out in next-to-leading order QCD at both fixed target and collider energies. We show that the differential cross section in the region $Q_{T} \geq Q / 2$ is dominated by subprocesses initiated by incident gluons. Dominance of the $q g$ contribution in the massive lepton-pair case is as strong if not stronger than it is in the prompt photon case. Massive lepton-pair differential cross sections are therefore an additional useful source of constraints 
on the gluon density. We compare calculations with data and provide predictions for the differential cross section as a function of $Q_{T}$ in proton-antiproton reactions at a center-ofmass energy of $1.8 \mathrm{TeV}$, in proton-nucleon reactions at laboratory momentum $800 \mathrm{GeV}$, and in proton-proton reactions at LHC energies.

As long $Q_{T}$ is large, the perturbative requirement of small $\alpha_{s}\left(Q_{T}\right)$ can be satisfied without a large value of $Q$. We therefore explore and advocate the potential advantages of studies of $d^{2} \sigma / d Q d Q_{T}$ as a function of $Q_{T}$ for modest values of $Q, Q \sim 2 \mathrm{GeV}$, below the range of the traditional Drell-Yan region.

As our calculations demonstrate, the $Q_{T}$ distribution of massive lepton pair production offers a valuable additional method for direct measurement of the gluon momentum distribution. This method makes good use of a significant data base at collider and fixed target energies. The method is similar in principle to the approach based on prompt photon production, but it avoids the experimental and theoretical complications of photon isolation that beset studies of prompt photon production. No isolation would seem necessary in the case of massive virtual photon production (and subsequent decay into a pair of muons) in typical collider or fixed target experiments. Muons are observed only after they have penetrated a substantial hadron absorber. Thus, hadrons within a typical cone about the direction of the $\gamma^{*}$ will have been stopped, and the massive lepton-pair signal will be entirely inclusive. For this reason, we claim that the cross section for massive lepton-pair production at large $Q_{T}$ is an advantageous source of information on the gluon density.

All-orders resummation is known to be important for the description of the $Q_{T}$ distribution of massive lepton-pair production at small and modest values of $q_{T}$. In this paper, we compare the resummed results with the purely perturbative results in order to try to establish the value above which we can be reasonably confident that fixed-order perturbation theory is adequate on its own. For the goals we have in mind, it would appear best to restrict attention to the region in $Q_{T}$ above the value at which the resummed result falls below the fixed-order perturbative expectation. A rough rule-of-thumb based on our calculations is $Q_{T} \geq Q / 2$. The published data [16] at fixed-target momenta do not extend into 
this domain. Their reach is exhausted at just about the value in $Q_{T}$ at which the fixed-order calculation should be reliable. To establish the utility of massive lepton-pair production at fixed-target momenta for extraction of the gluon density, it would be valuable to obtain data in the traditional Drell-Yan region above $Q \simeq 4.0 \mathrm{GeV}$ but whose reach in $Q_{T}$ extends into the fixed-order perturbative domain. The experimental rates at the $p \bar{p}$ collider energy of $\sqrt{S}=1.8 \mathrm{TeV}$ should be adequate over a wide range in $Q_{T}$.

\section{ACKNOWLEDGMENTS}

Work in the High Energy Physics Division at Argonne National Laboratory is supported by the U.S. Department of Energy, Division of High Energy Physics, Contract W-31-109ENG-38. We have benefitted from communications with C. Brown, G. T. Garvey, and J. Moss concerning their data from FNAL fixed-target experiments and are grateful to P. Arnold, R. Kauffman, and M. H. Reno for providing copies of their numerical codes. 


\section{REFERENCES}

[1] S. Drell and T. M. Yan, Phys. Rev. Lett. 25, 316 (1970); Ann. Phys. (NY) 66, 578 (1971).

[2] E. L. Berger and J.-W. Qiu, Phys. Lett. B248, 371 (1990) and Phys. Rev. D44, 2002 (1991).

[3] H. Baer, J. Ohnemus, and J. F. Owens, Phys. Rev. D42, 61 (1990).

[4] P. Aurenche et al, Nucl. Phys. B399, 34 (1993).

[5] L. E. Gordon and W. Vogelsang, Phys. Rev. D48, 3136 (1993) and D50, 1901 (1994);

M. Glück, L. E. Gordon, E. Reya, and W. Vogelsang, Phys. Rev. Lett. 73, 388 (1994);

L. E. Gordon, Nucl. Phys. B501, 175 (1997).

[6] P. B. Arnold and M. H. Reno, Nucl. Phys. B319, 37 (1989), and erratum Nucl. Phys. B330, 284 (1990); R. J. Gonsalves, J. Pawlowski, and C.-F. Wai, Phys. Rev. D40, 2245 (1989).

[7] J. Collins, D. Soper, and G. Sterman, Nucl. Phys. B250, 199 (1985); J. Collins and D. Soper, Nucl. Phys. B193, 381 (1981), Nucl. Phys. B197, 446 (1982), and erratum Nucl. Phys. B213, 545 (1983).

[8] C. Davies, B. Webber, and W. J. Stirling, Nucl. Phys. B256, 413 (1985); C. Davies and W. J. Stirling, Nucl. Phys. B244, 337 (1984).

[9] G. Altarelli, R. K. Ellis, M. Greco, and G. Martinelli, Nucl. Phys. B246, 12 (1984).

For a recent treatment and list of references, see R. K. Ellis, D. A. Ross, and S. Veseli, Nucl. Phys. B503, 309 (1997).

[10] P. B. Arnold and R. Kauffman, Nucl. Phys. B349, 381 (1991).

[11] G. A. Ladinsky and C. P. Yuan, Phys. Rev. D50, 4239 (1994).

[12] E. L. Berger and D. E. Soper, Nucl. Phys. B247, 29 (1984). 
[13] F. Halzen and D. M. Scott, Phys. Lett. 80B, 410 (1979).

[14] P. Aurenche, R. Baier, and M. Fontannaz, Phys. Lett. B209, 375 (1988).

[15] E. L. Berger, X.-F. Guo, and J.-W. Qiu, Phys. Rev. Lett. 76, 2234 (1996); Phys. Rev. D54, 5470 (1996). E. L. Berger, X.-F. Guo, and J.-W. Qiu, in '97 QCD and High Energy Hadronic Interactions, Proceedings of the XXXIInd Rencontres de Moriond, Les Arcs, France, March, 1997, edited by J. Tran Thanh Van (Editions Frontieres, Paris, 1997) pp $267-274$.

[16] Fermilab E772 Collaboration, P. L. McGaughey et al, Phys. Rev. D50, 3038 (1994).

[17] CERN UA1 Collaboration, C. Albajar et al, Phys. Lett. B209, 397 (1988).

[18] S. Chang, C. Coriano, R. D. Field, and L. E. Gordon, Argonne report ANL-HEP-PR97-12, Phys. Rev. D (to appear).

[19] R. K. Ellis, G. Martinelli and R. Petronzio, Nucl. Phys. B211, 106 (1983).

[20] M. Klasen, G. Kramer, and B. Pötter, Eur.Phys.J. C1, 261 (1998).

[21] H.L. Lai et al, CTEQ Collaboration, Phys. Rev. D55, 1280 (1997).

[22] The normalization of the published data seems incorrect. We have multiplied the published cross sections by a factor 3.7, consistent with information provided to us by J. Moss (private communication).

[23] CDF Collaboration, F. Abe et al, Phys. Rev. D48, 2998 (1993); Phys. Rev. Lett. 73, 2662 (1994); D0 Collaboration, S. Abachi et al, Phys. Rev. Lett. 77, 5011 (1996). 


\section{FIGURES}
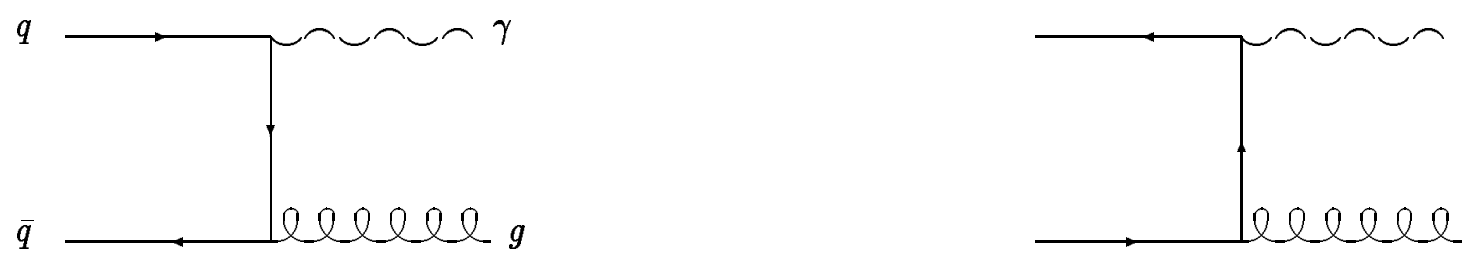

(a)
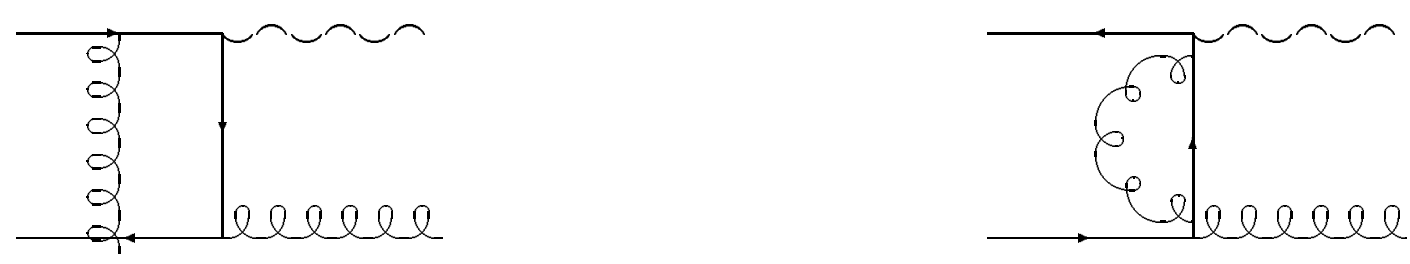

(b)
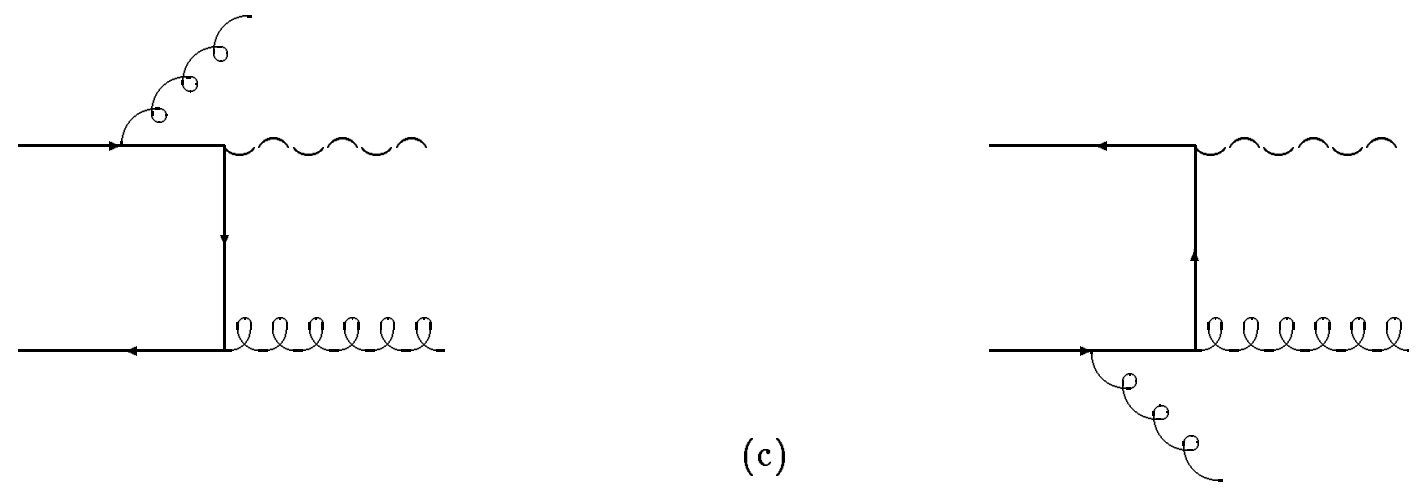

FIG. 1. (a)Lowest-order Feynman diagrams for the direct process $q+\bar{q} \rightarrow \gamma+g$. (b) Examples of virtual gluon loop diagrams. (c) Examples of next-to-leading order three-body final-state diagrams. 

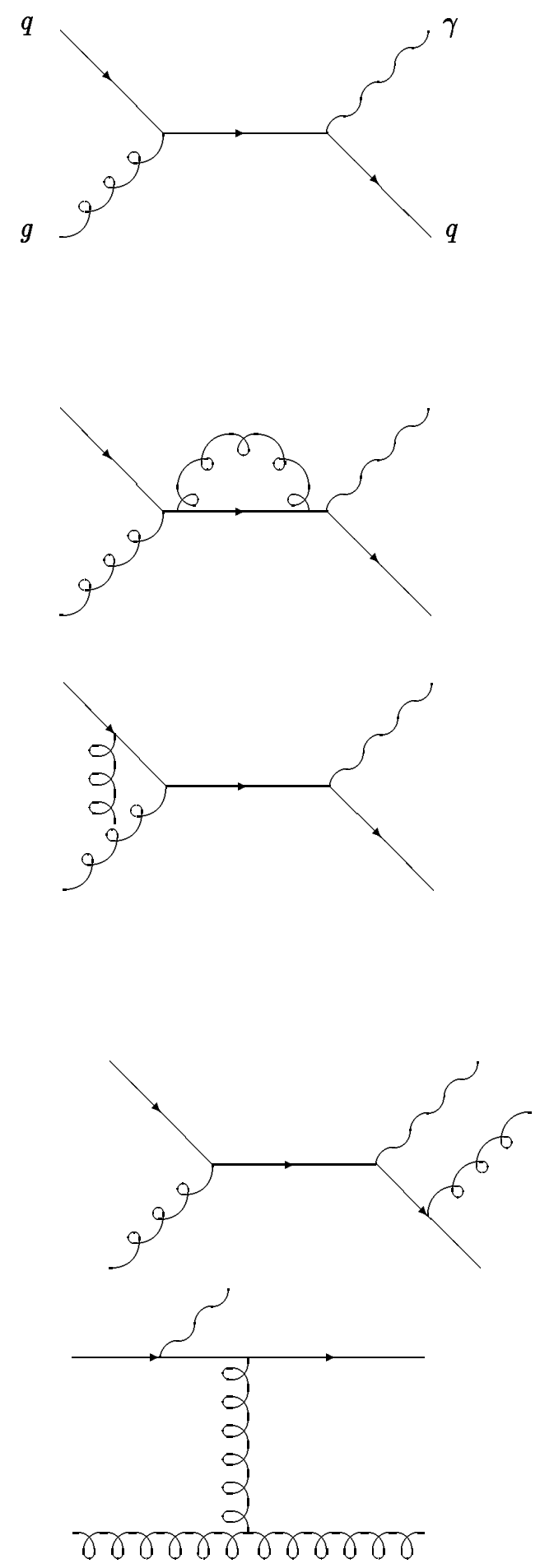

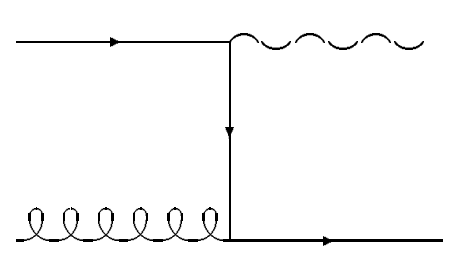

(a)
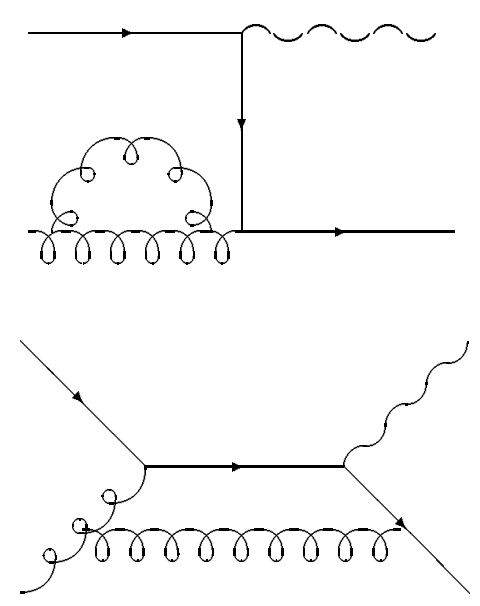

(b)

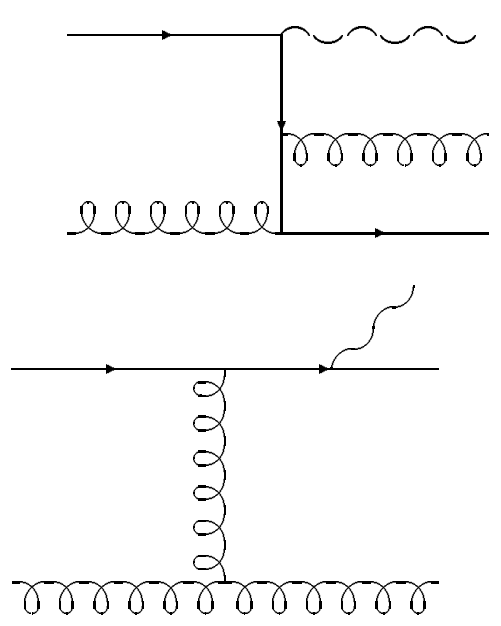

(c)

FIG. 2. As in Fig. 1, but for the subprocesses initiated by the $q+g$ initial state. 

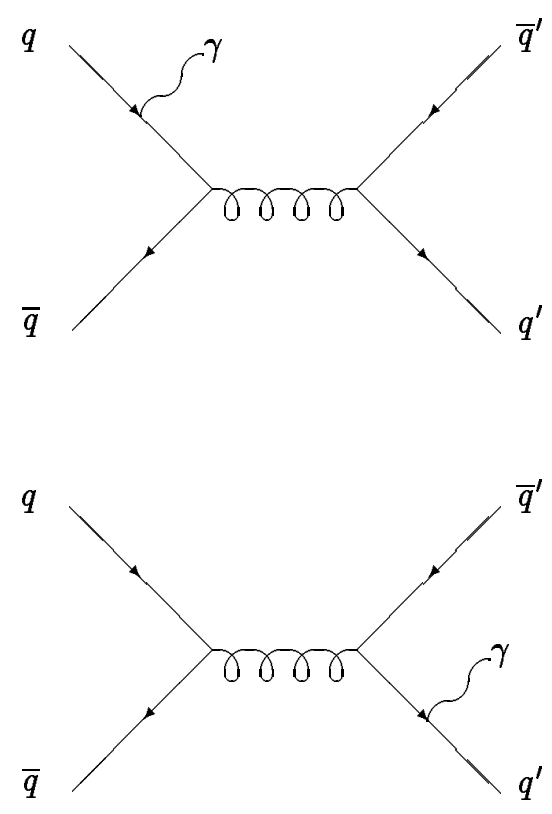

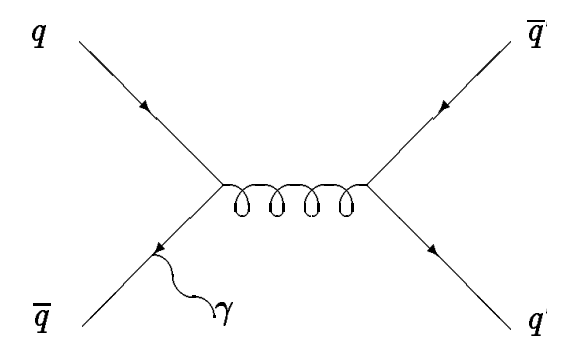

(a)

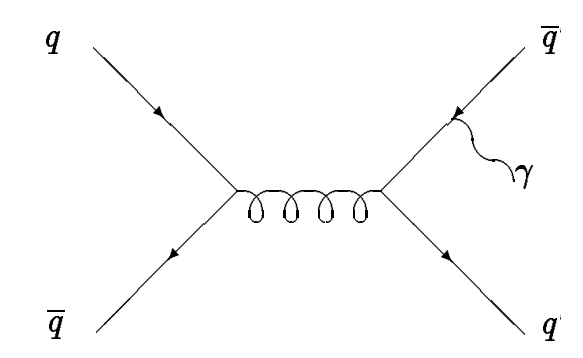

(b)

FIG. 3. Diagrams that illustrate situations in which the photon becomes collinear to a quark in (a) the initial state and (b) the final state. 


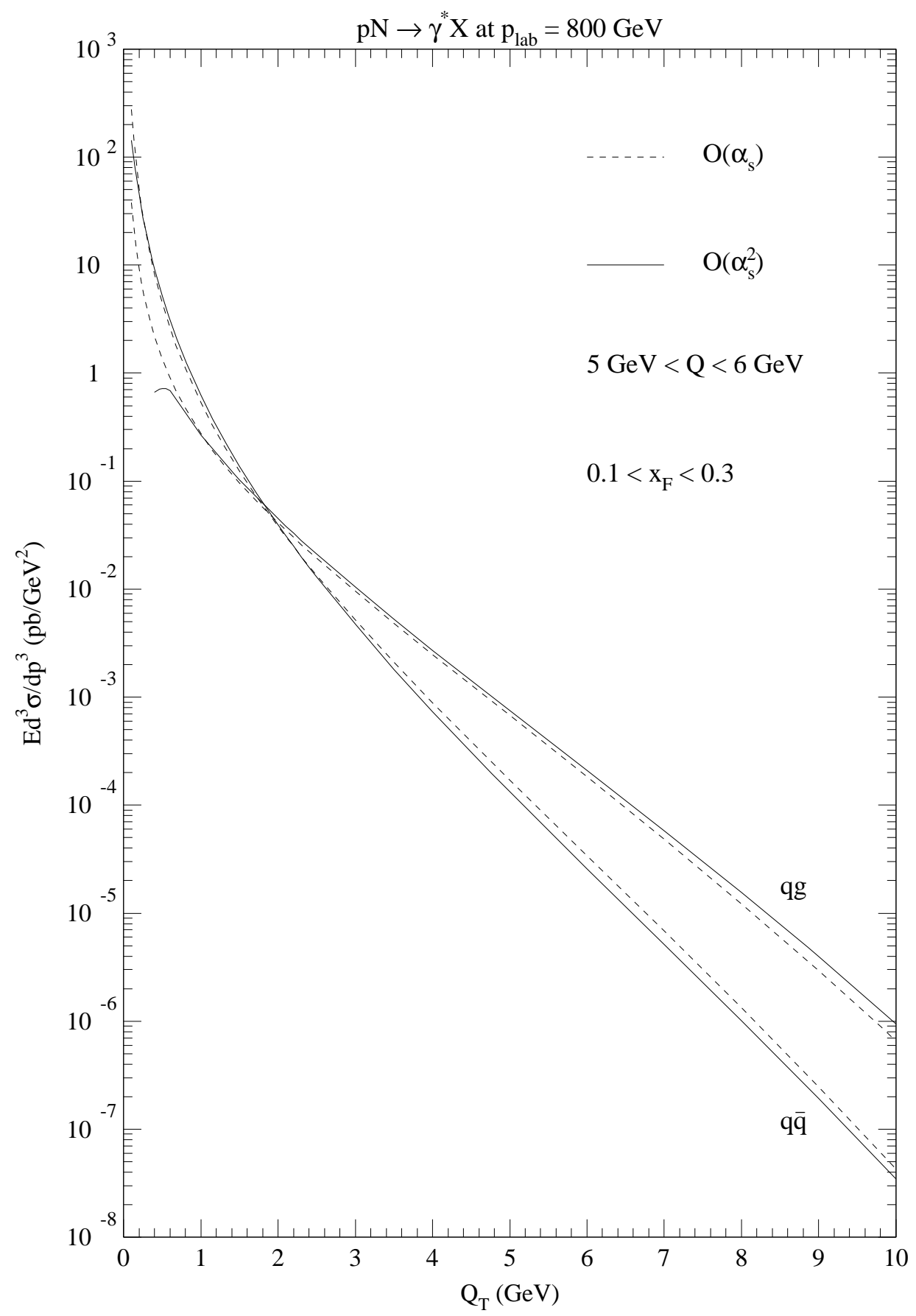

FIG. 4. Lowest order $\mathcal{O}\left(\alpha_{s}\right)$ (dashed lines) and next-to-leading order $\mathcal{O}\left(\alpha_{s}^{2}\right)$ (solid lines) perturbative calculations of the invariant inclusive cross section $E d^{3} \sigma / d p^{3}$ as a function of $Q_{T}$ for $p N \rightarrow \gamma^{*} X$ at $p_{\text {lab }}=800 \mathrm{GeV}$, in the $\overline{\mathrm{MS}}$ scheme. Contributions from the $q g$ and $q \bar{q}$ channels are shown separately. The results are integrated over the scaled longitudinal momentum interval 0.1 $<x_{F}<0.3$ and over the interval $5.0<Q<6.0 \mathrm{GeV}$, and they are divided by the bin width in $x_{F}$. 


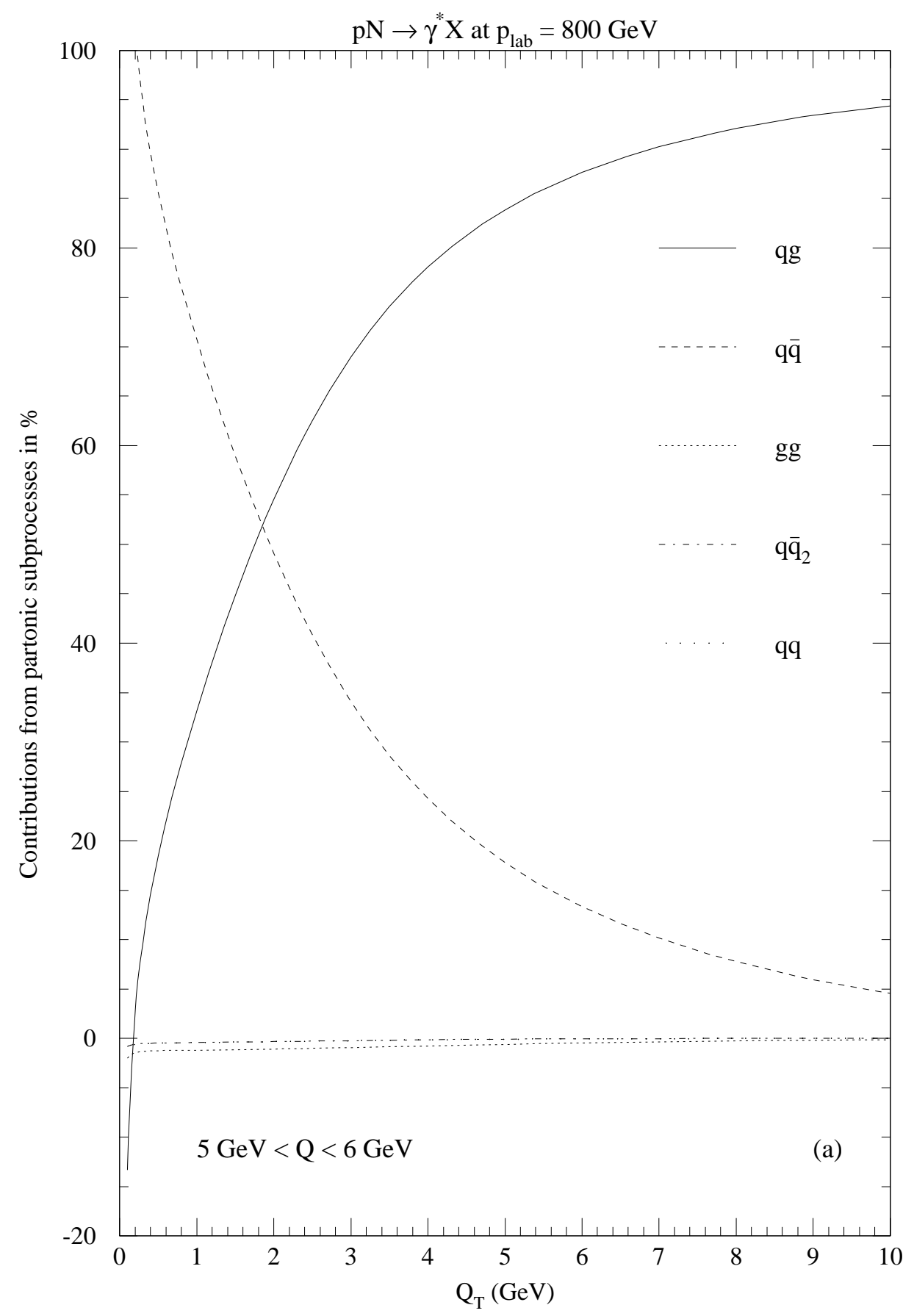




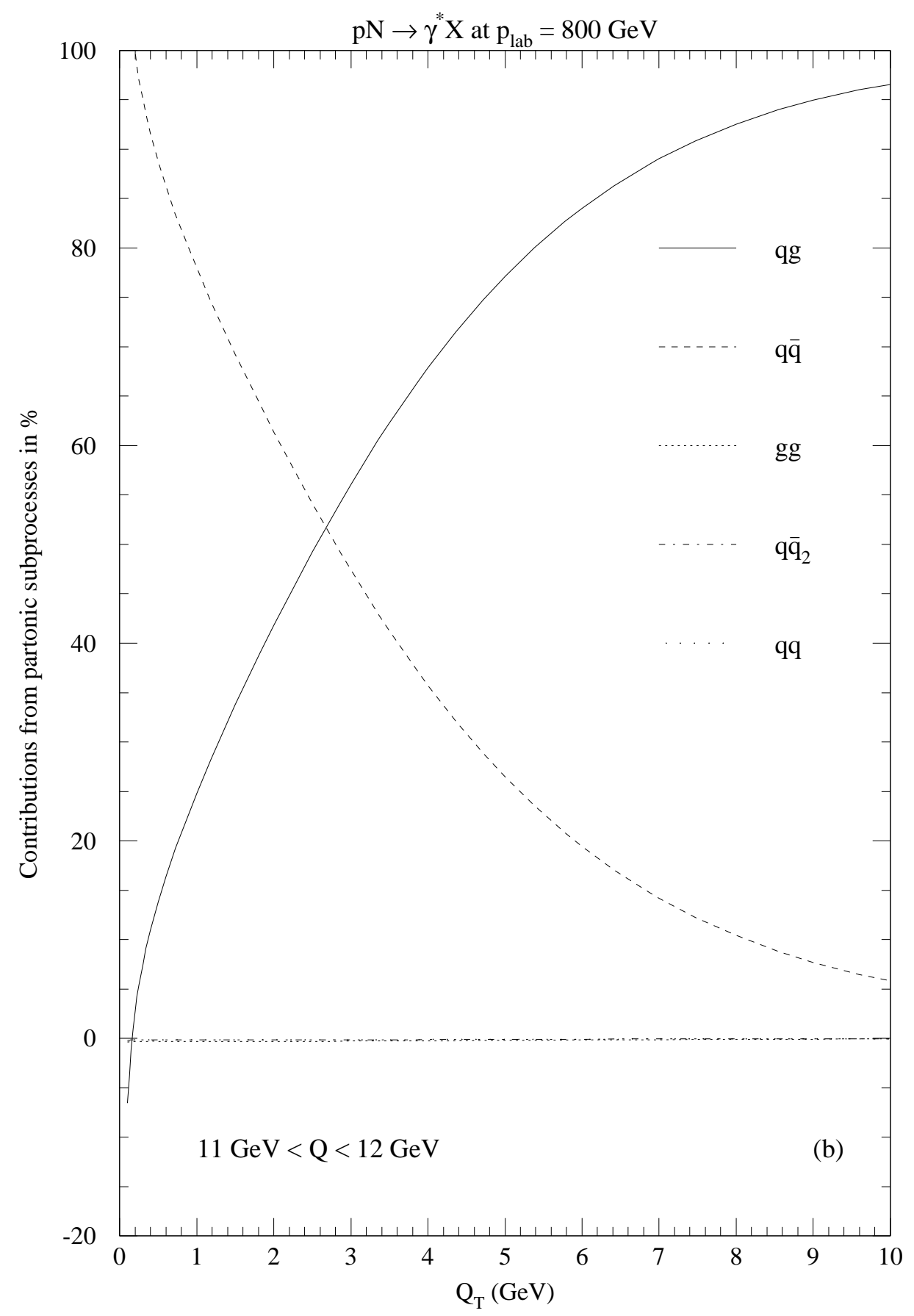




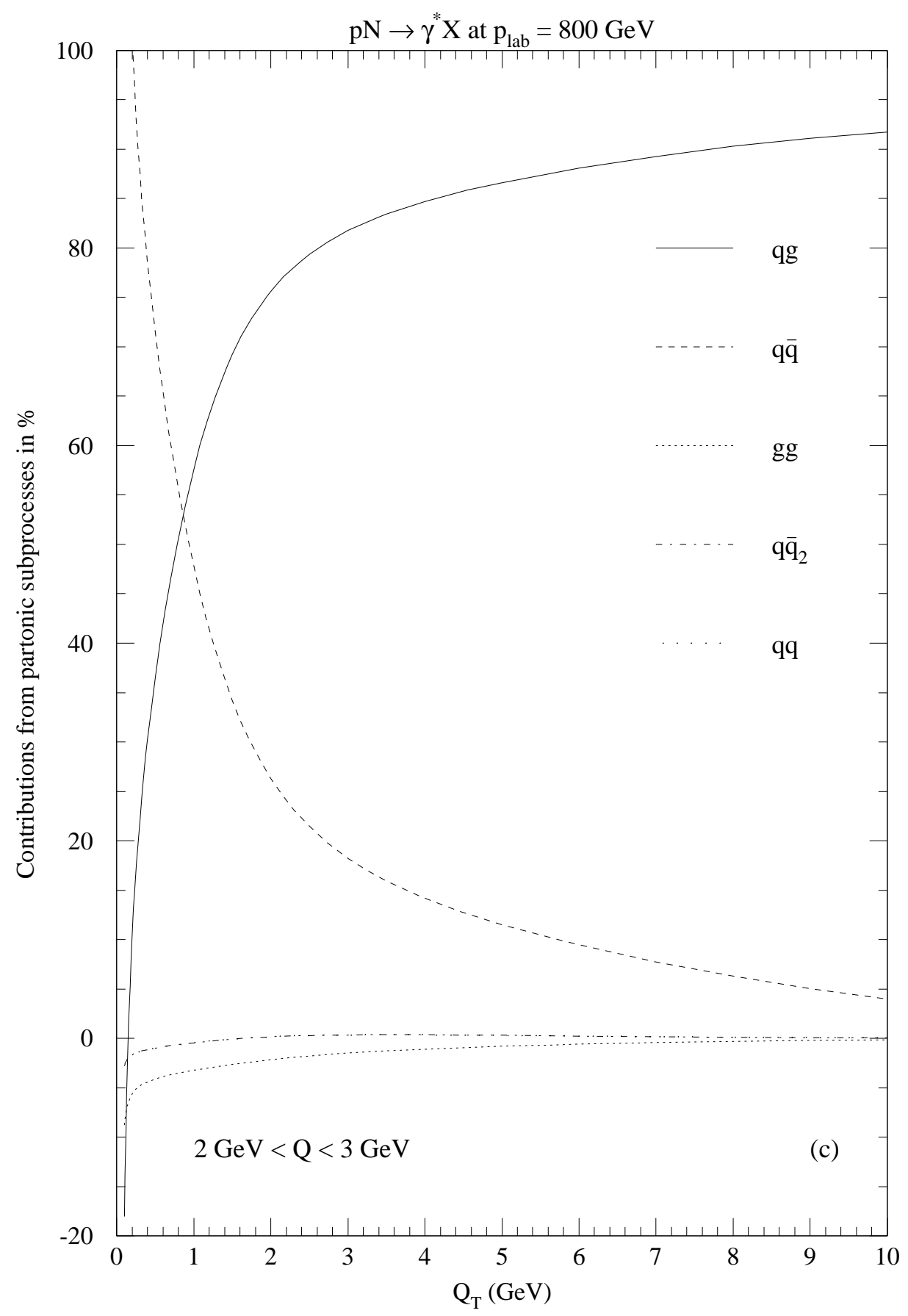

FIG. 5. Contributions from the various partonic subprocesses to the invariant inclusive cross section $E d^{3} \sigma / d p^{3}$ as a function of $Q_{T}$ for $p N \rightarrow \gamma^{*} X$ at $p_{\text {lab }}=800 \mathrm{GeV}$. The cross section is integrated over the scaled longitudinal momentum interval $0.1<x_{F}<0.3$ and over the intervals (a) $5.0<Q<6.0 \mathrm{GeV}$, (b) $11.0<Q<12.0 \mathrm{GeV}$, and (c) $2.0<Q<3.0 \mathrm{GeV}$, and divided by the bin width in $x_{F}$. The contributions are labeled by $q g$ (solid), $q \bar{q}$ (dashed), $g g$ (dotted), $q \bar{q}_{2}$ non-factorizable parts (dot-dashed), and $q q$ (wide dots). The $q q$ and $q \bar{q}_{2}$ results nearly coincide. 


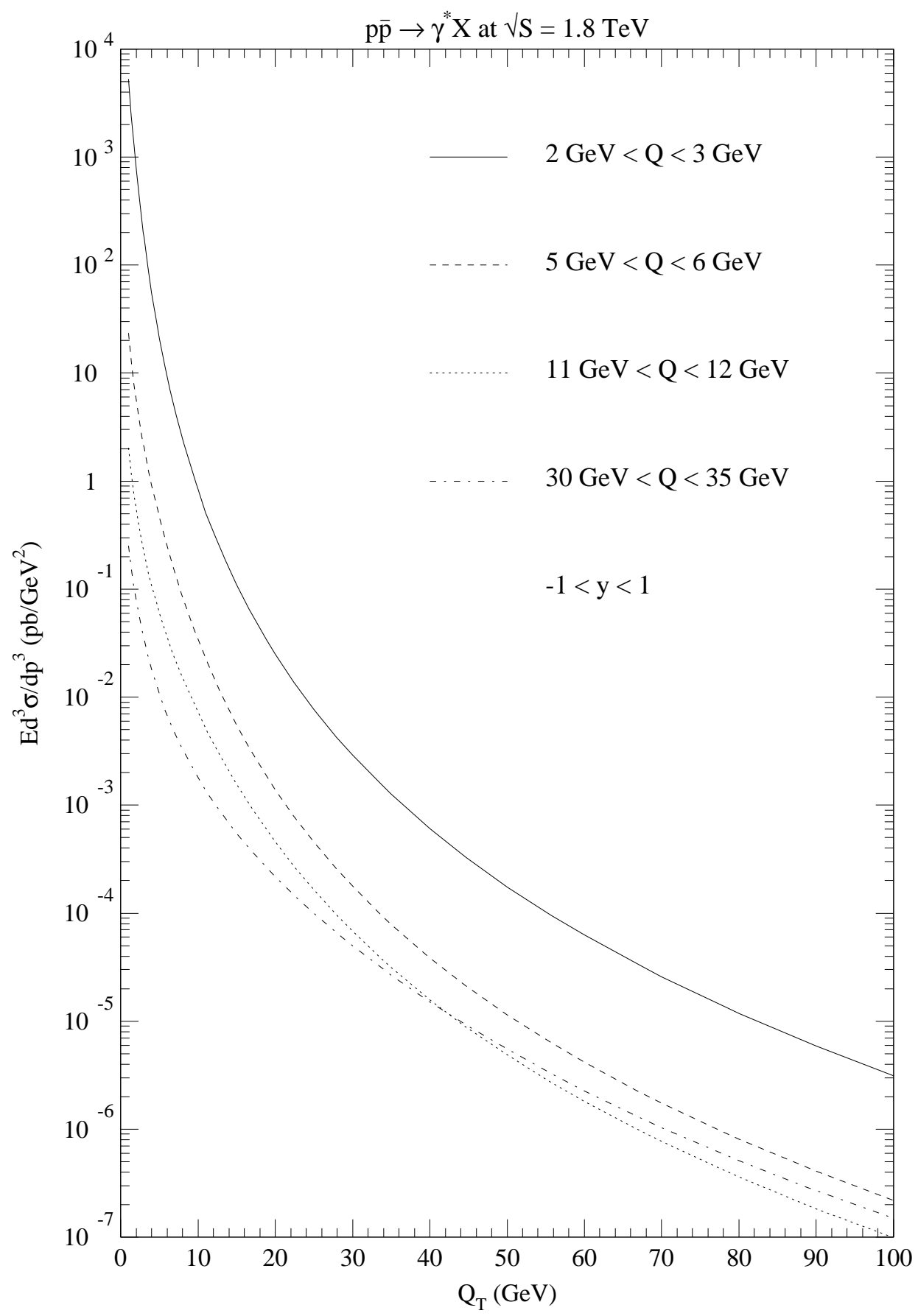

FIG. 6. Differential cross sections $E d^{3} \sigma / d p^{3}$ as a function of $Q_{T}$ for $p \bar{p} \rightarrow \gamma^{*} X$ at $\sqrt{S}=1.8$ TeV integrated over the rapidity interval $-1.0<y<1.0$ and over the intervals $2.0<Q<3.0 \mathrm{GeV}$ (solid), $5.0<Q<6.0 \mathrm{GeV}$ (dashed), $11.0<Q<12.0 \mathrm{GeV}$ (dotted), and $30.0<Q<35.0 \mathrm{GeV}$ (dot-dashed). The results of the integration have been divided by the bin width in $y$. 


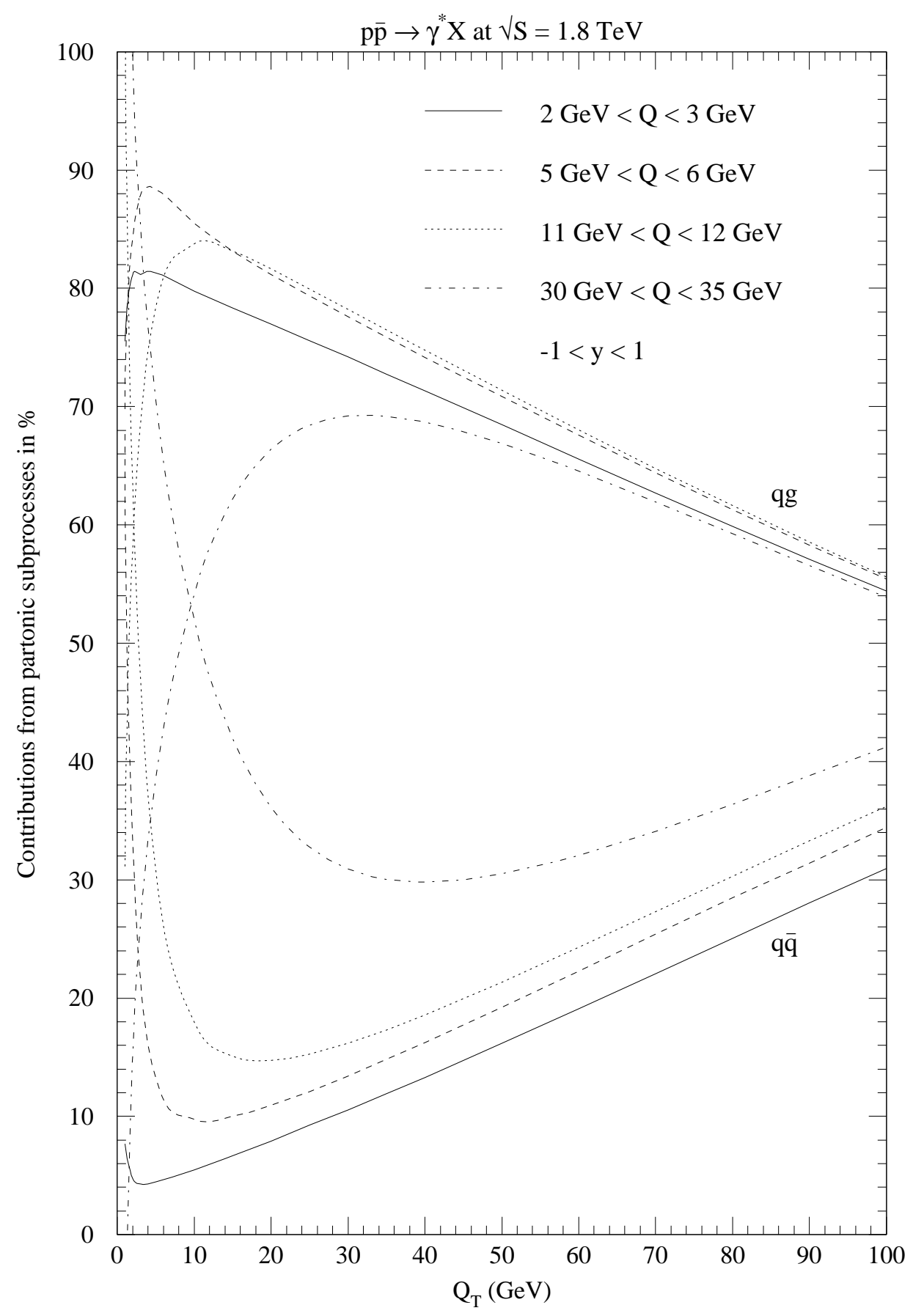

FIG. 7. Contributions from the partonic subprocesses $q g$ and $q \bar{q}$ to the invariant inclusive cross section $E d^{3} \sigma / d p^{3}$ as a function of $Q_{T}$ for $p \bar{p} \rightarrow \gamma^{*} X$ at $\sqrt{S}=1.8 \mathrm{TeV}$. The cross section is integrated over the rapidity interval $-1.0<y<1.0$ and over the intervals $2.0<Q<3.0 \mathrm{GeV}$ (solid), $5.0<Q<6.0 \mathrm{GeV}$ (dashed), $11.0<Q<12.0 \mathrm{GeV}$ (dotted), and $30.0<Q<35.0 \mathrm{GeV}$ (dot-dashed). The results of the integration have been divided by the bin width in $y$. 


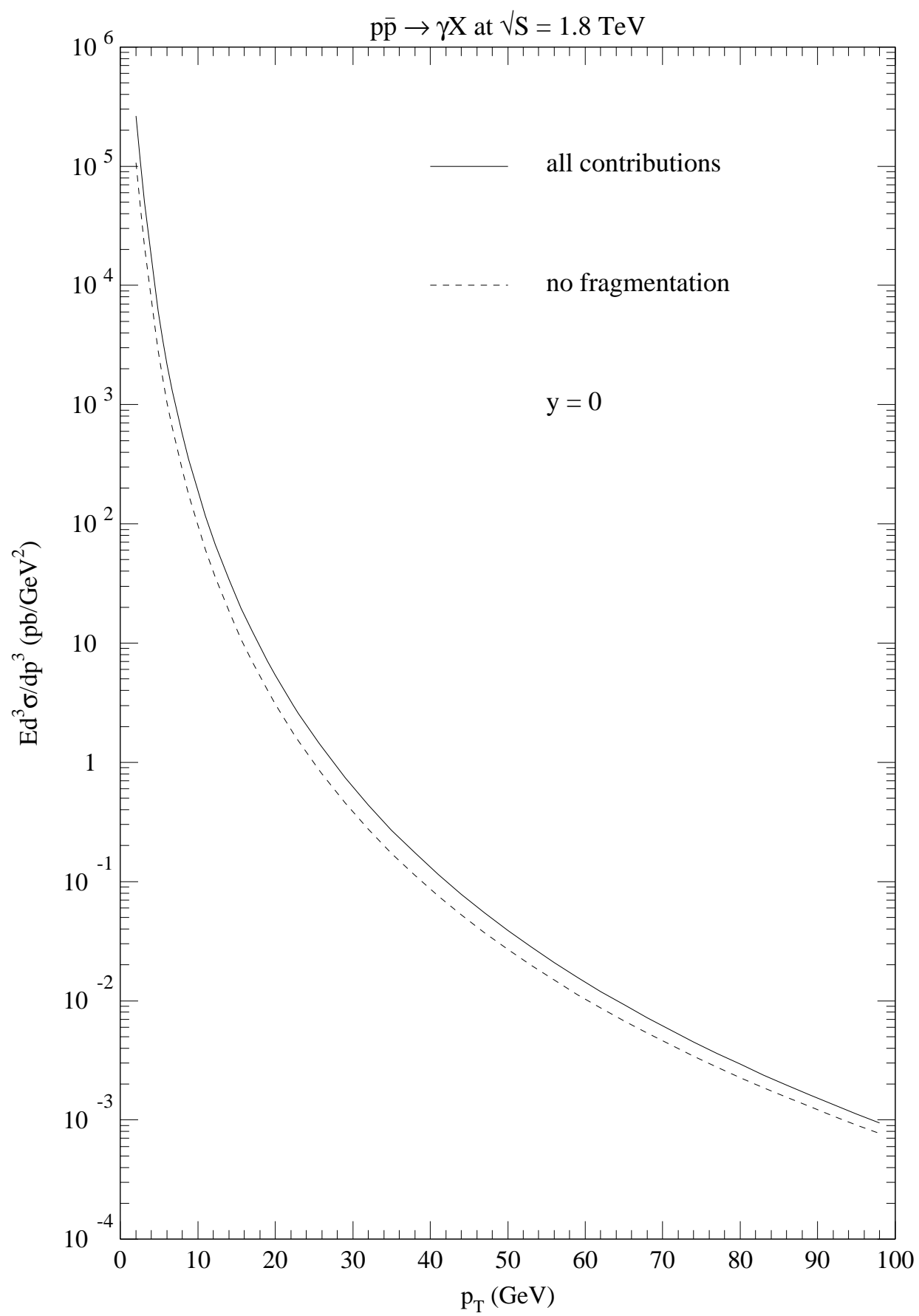

FIG. 8. Differential cross sections $E d^{3} \sigma / d p^{3}$ as a function of $p_{T}$ for real photon production $p \bar{p} \rightarrow \gamma X$ at $\sqrt{S}=1.8 \mathrm{TeV}$ in the $\overline{\mathrm{MS}}$-scheme for two cases: no fragmentation terms included (dashed) and the inclusive case with full fragmentation included (solid). The rapidity $y=0$. 


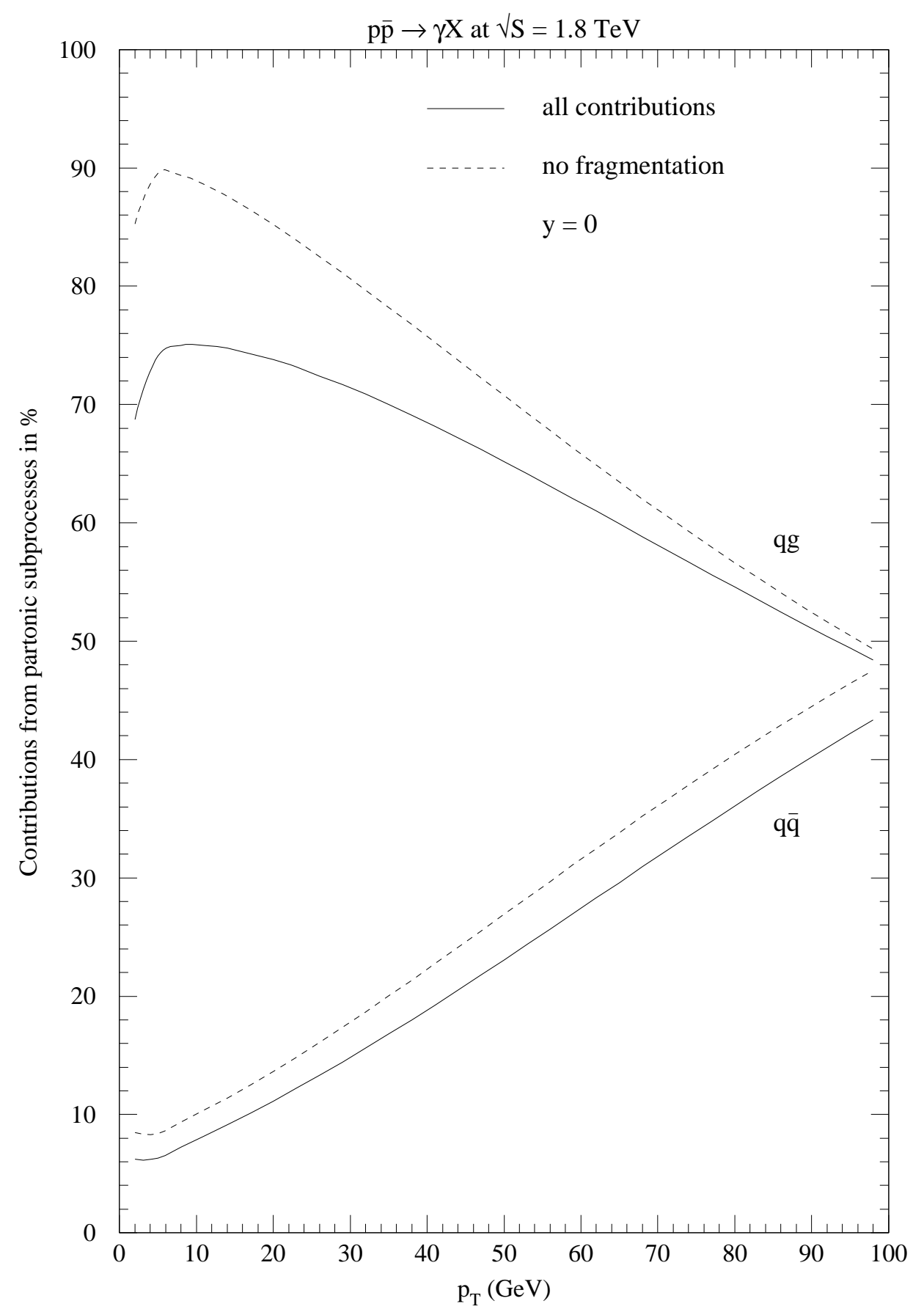

FIG. 9. Separate contributions from the $q \bar{q}$ and the $q g$ channels to the differential cross sections $E d^{3} \sigma / d p^{3}$ as a function of $p_{T}$ for real photon production $p \bar{p} \rightarrow \gamma X$ at $\sqrt{S}=1.8 \mathrm{TeV}$ in the $\overline{\mathrm{MS}}$-scheme for two cases: no fragmentation terms included (dashed lines) and the inclusive case with full fragmentation included (solid lines). The rapidity $y=0$. 


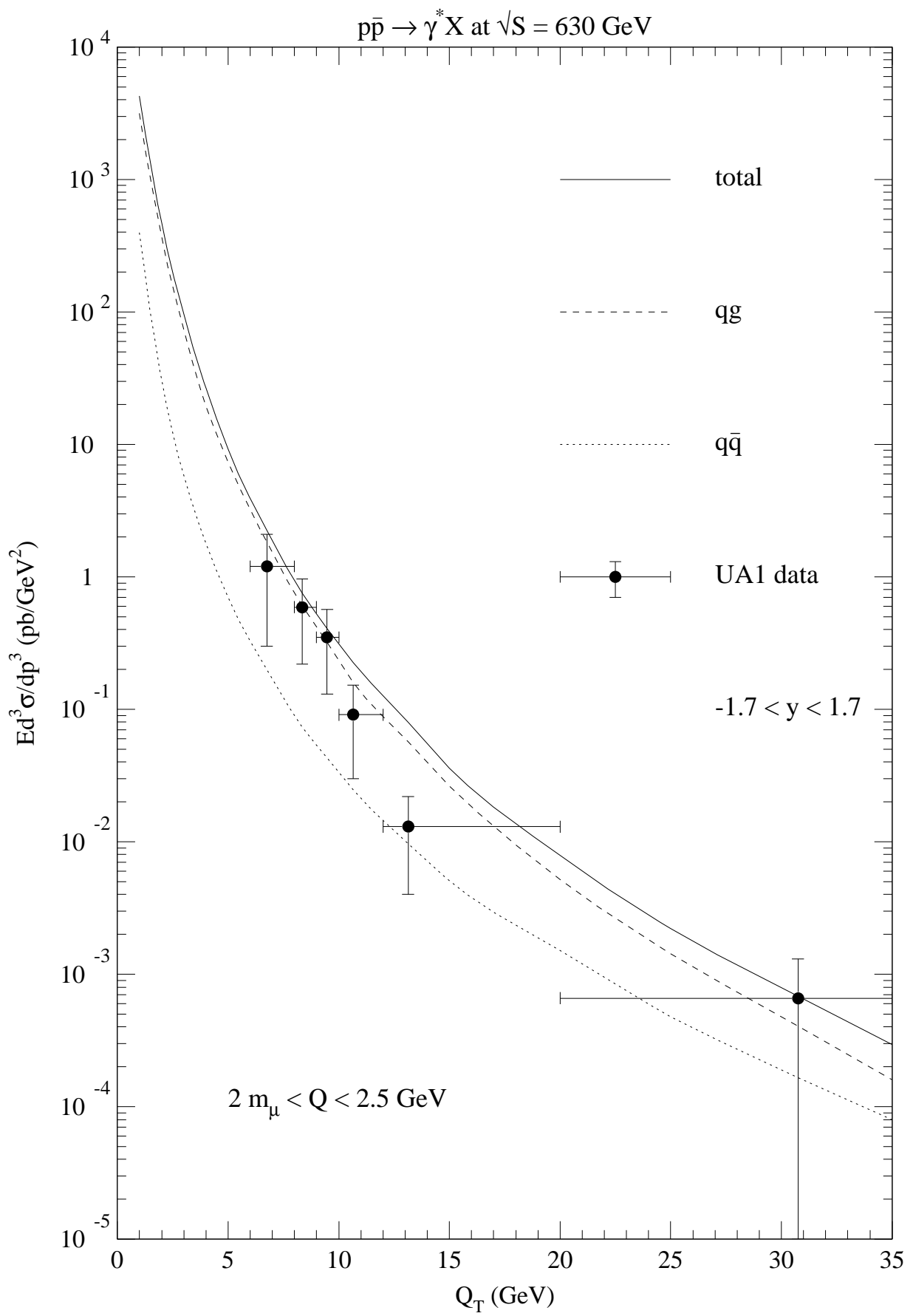

FIG. 10. Invariant inclusive cross section $E d^{3} \sigma / d p^{3}$ as a function of $Q_{T}$ for $p \bar{p} \rightarrow \gamma^{*}+X$ at $\sqrt{S}=630 \mathrm{GeV}$, with $2 m_{\mu} \leq Q \leq 2.5 \mathrm{GeV}$, and averaged over the rapidity interval $-1.7<y<1.7$. The next-to-leading order perturbative cross section (solid) is shown along with its two major components, the $q g$ (dashed) and $q \bar{q}$ (dotted) contributions. The data are from the CERN UA1 collaboration. 


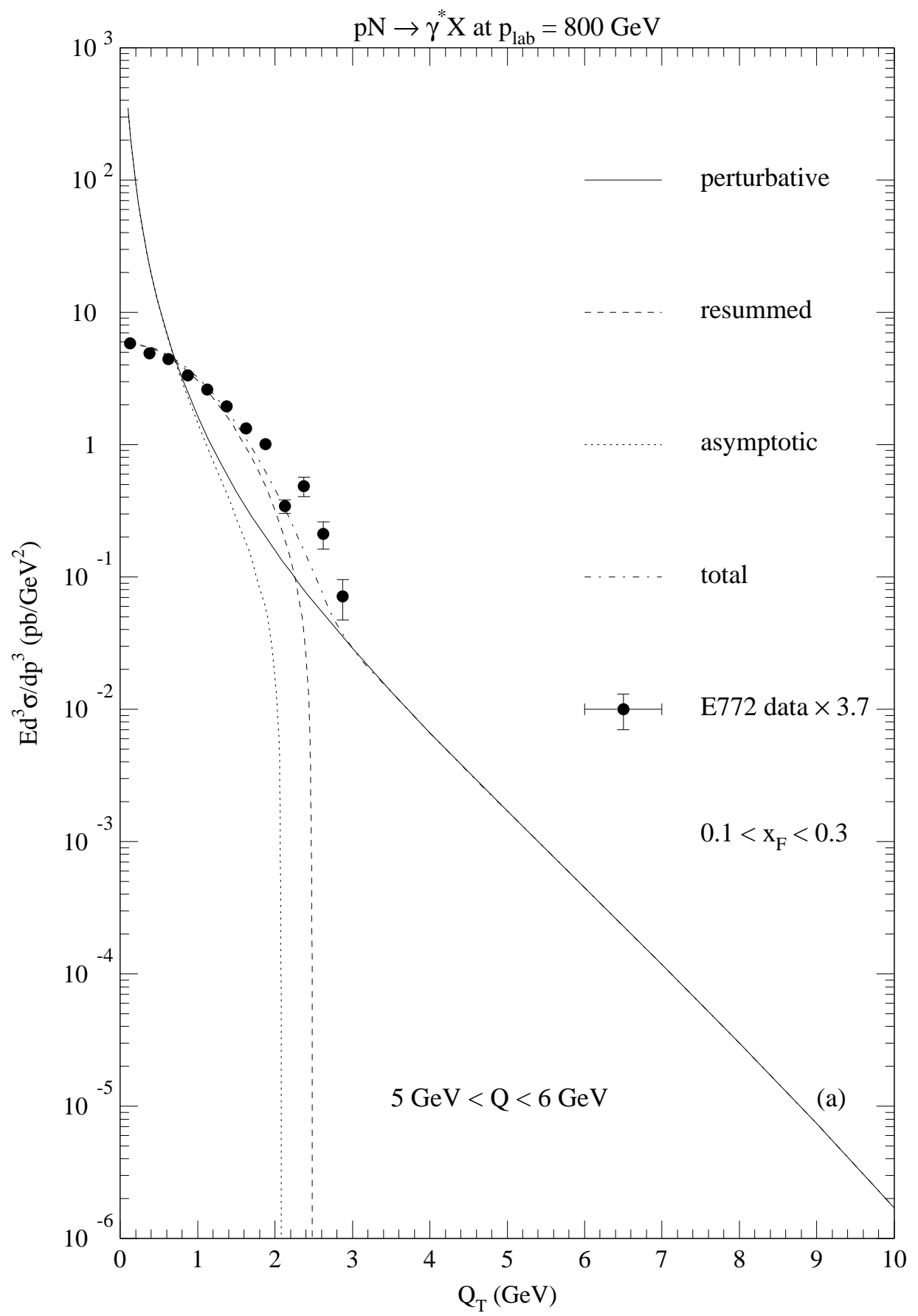




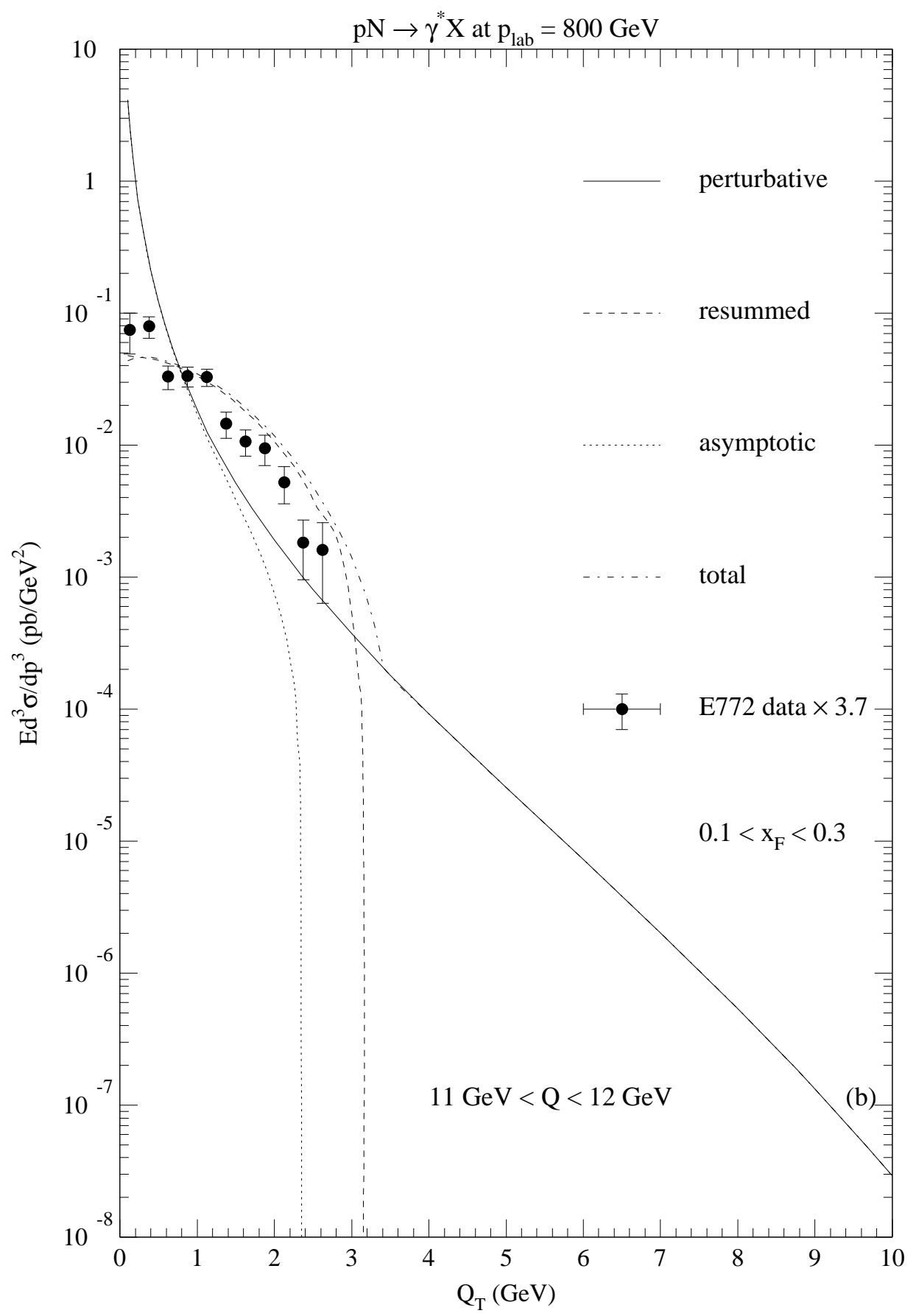




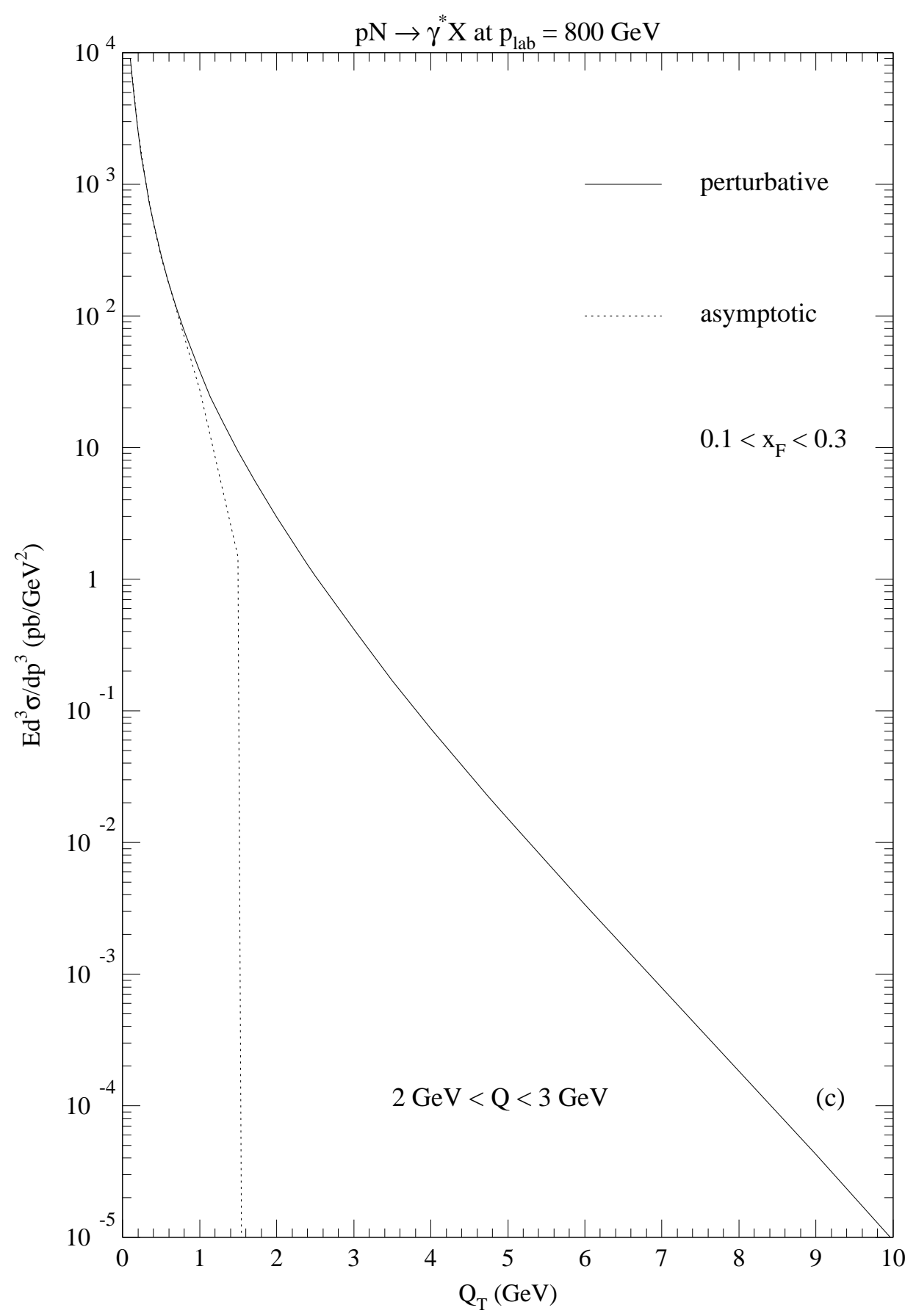

FIG. 11. Invariant inclusive cross section $E d^{3} \sigma / d p^{3}$ as a function of $Q_{T}$ for $p N \rightarrow \gamma^{*}+X$ at $p_{\text {lab }}=800 \mathrm{GeV}$, averaged over the scaled longitudinal momentum interval $0.1<x_{F}<0.3$. The next-to-leading order perturbative cross section (solid) is shown along with the all-orders resummed expectation (dashed), the asymptotic result (dotted), and a matched expression (dot-dashed). The data are from the Fermilab E772 collaboration. (a) $5.0<Q<6.0 \mathrm{GeV}$, (b) $11.0<Q<12.0 \mathrm{GeV}$, and (c) $2.0<Q<3.0 \mathrm{GeV}$. 


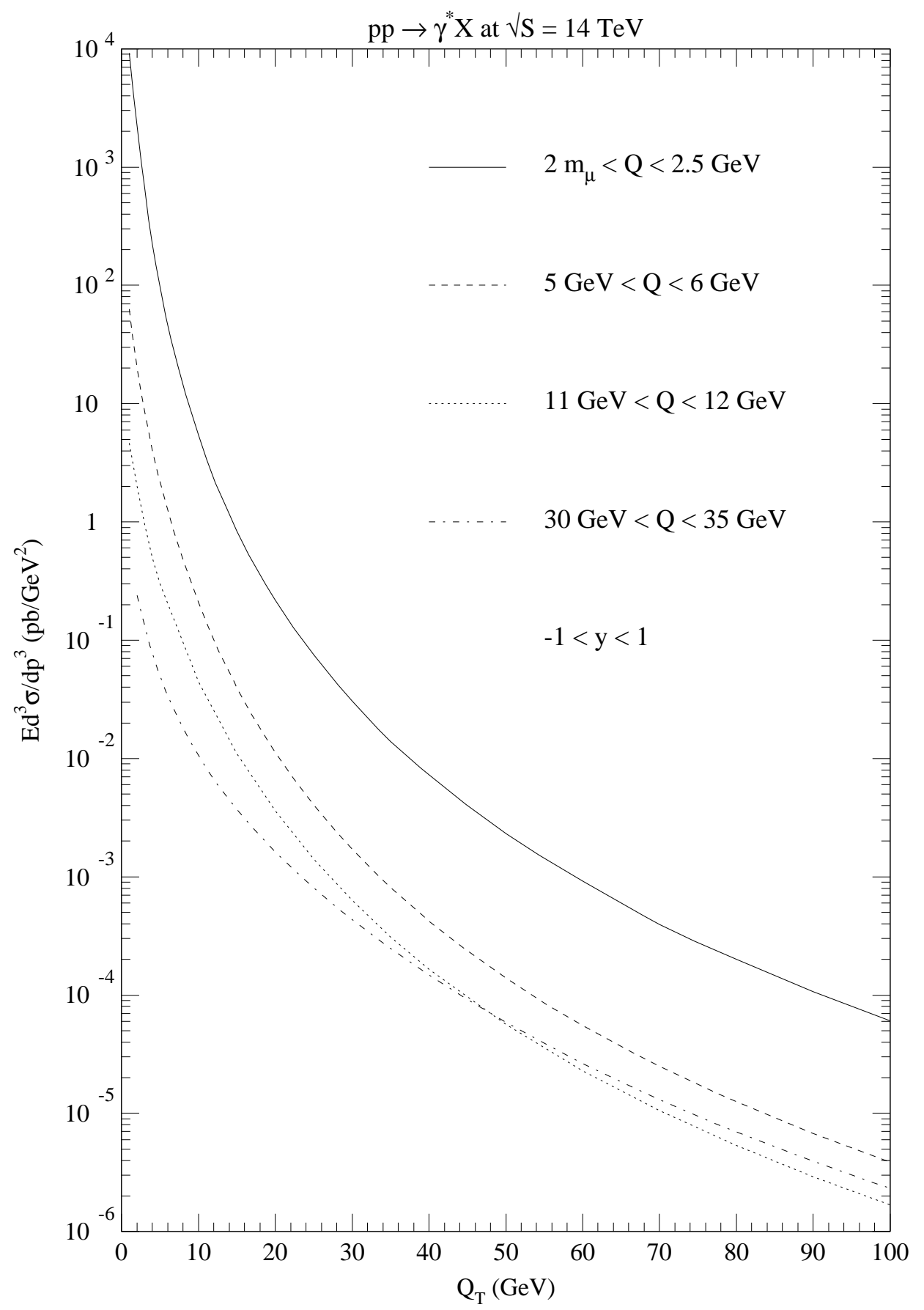

FIG. 12. Invariant inclusive cross section $E d^{3} \sigma / d p^{3}$ as a function of $Q_{T}$ for $p p \rightarrow \gamma^{*}+X$ at $\sqrt{S}=14 \mathrm{TeV}$ averaged over the rapidity interval $-1.0<y<1.0$. The four curves show the next-to-leading order perturbative predictions integrated over 4 different intervals of Q: $2 m_{\mu}<Q<$ $2.5 \mathrm{GeV}, 5.0<Q<6.0 \mathrm{GeV}, 11.0<Q<12.0 \mathrm{GeV}$, and $30.0<Q<35.0 \mathrm{GeV}$. 